Die Forschung hat bisher vor allem die Großbanken im Nationalsozialismus ins Visier genommen. Sparkassen und Regionalbanken wurden dagegen weitgehend ignoriert, obwohl sie auch damals beträchtliche Teile des Geldgeschäftes abwickelten. Horst Möller, der langjährige Direktor des Instituts für Zeitgeschichte, beschreitet mit seiner Untersuchung der Bayerischen Vereinsbank 1933 bis 1945 ganz neue Wege. Das Ergebnis ist frappierend: Der Bayerischen Vereinsbank, einer der größten bayerischen Regionalbanken, gelang es bemerkenswert lange, ihre Autonomie weitgehend zu bewahren und sich dem Gleichschaltungsdruck des NS-Regimes zu entziehen. Maßgebend dafür waren bis 1938 der Ruhrindustrielle Paul Reusch und die jüdische Mendelssohn-Bank, die als Minderheitsaktionäre Klima und Kultur der Bank mitprägten, und nach ihrer Verdrängung couragierte Bankfachleute, die noch im Krieg auf ein gewisses $\mathrm{Ma} ß$ an Eigenständigkeit pochten - nicht erfolglos, wie Horst Möller zeigen kann.

Horst Möller

\title{
Die Bayerische Vereinsbank zwischen Resistenz und Gleichschaltung 1933-1945
}

\section{Banken im NS-Regime als zeitgeschichtliches Forschungsthema}

Die Geschichte von Unternehmen, Banken, Ministerien und Behörden zur Zeit der nationalsozialistischen Diktatur hat Konjunktur. Dies ist insofern nicht überraschend, als während der letzten zwei Jahrzehnte nach und nach fast alle Berufsgruppen und Wirtschaftssektoren ins Blickfeld der Geschichtsschreibung geraten sind. Hierbei handelte es sich einerseits um wissenschaftsimmanente Entwicklungen, die aus einer sich mehr und mehr differenzierenden Forschung erwach$\operatorname{sen}^{1}$, andererseits um die in der deutschen Öffentlichkeit seit vielen Jahrzehnten immer wieder diskutierte Frage nach der Verantwortlichkeit für die und in der nationalsozialistischen Diktatur.

Wie frei war ein Unternehmen in der traditionsgeleiteten Weiterentwicklung seiner Unternehmenskultur und seiner geschäftlichen Aktivitäten, wie fremdbe-

\footnotetext{
${ }^{1}$ Den besten Forschungsüberblick bieten Klaus Hildebrand, Das Dritte Reich, 6., neubearb. Aufl., München 2003, sowie Ian Kershaw, Der NS-Staat. Geschichtsinterpretationen und Kontroversen im Überblick, 3., erw. Aufl., Hamburg 2002. - Dieser Aufsatz steht im Zusammenhang einer umfangreichen Studie des Verfassers: „Regionalbanken im Dritten Reich. Bayerische Hypotheken- und Wechsel-Bank, Bayerische Vereinsbank, Vereinsbank in Hamburg, Bayerische Staatsbank", die 2015 veröffentlicht wird. Die hier behandelte Thematik stellt einen Teilaspekt dar. Andere Themen, etwa die Frage, wieweit die Bank an „Arisierungen“ beteiligt war, werden in der o.g. Studie untersucht. Ich danke der HypoVereinsbank - UniCredit Bank AG München sowie ihrem Archiv, insbesondere seiner Leiterin, Frau Dipl. Hist. Elke Pfnür, für die uneingeschränkte Einsichtnahme in die Archivalien und die nachhaltige Unterstützung der Studie.
} 
stimmt war es unter den spezifischen Bedingungen des nationalsozialistischen Regimes? Wie tief waren Banken in das NS-Regime verstrickt? Welchen Nutzen hatten sie gegebenenfalls davon? Gibt es Besonderheiten für die Regionalbanken, welche Unterschiede bestehen zwischen ihnen und den Großbanken?

Die Forschung ist sich, ungeachtet zahlreicher Desiderate in Bezug auf Sparkassen, Genossenschaftsbanken, mittlere und kleinere Banken, über einige grundlegende Einschätzungen weitgehend einig: Es ist notwendig, das Agieren der Unternehmen und Banken im Kontext der Zeit und unter den Bedingungen der nationalsozialistischen Diktatur zu analysieren und zu bewerten. Die Banken und andere Unternehmen waren nicht autonome Akteure, sondern von der Politik des Regimes abhängig, es herrschte „ein weitgehender Primat der Politik“ gegenüber der Wirtschaft, das „Unternehmertum [hatte] keine Stimme in der Zielsetzung des NS-Regimes. Alle grundsätzlichen Entscheidungen wurden von einer politischen Führung gefällt, die die Wirtschaft als bloßes Werkzeug für die Verwirklichung von weitgesteckten ideologisch bestimmten Zielen betrachtete, deren volle Radikalität nur langsam aufgedeckt wurde.“ Doch haben „die deutschen Unternehmer sich schnell mit dem Dritten Reich abgefunden“2, zum Widerstand gehörte kaum ein Wirtschaftsführer, von Ausnahmen wie Robert Bosch abgesehen ${ }^{3}$.

Ebenso notwendig ist eine zeitliche Differenzierung, machte sich doch ab 1936/37 ein gewisses Unbehagen in den Unternehmensleitungen breit. Maßgebend dafür waren nicht nur die durchaus arbeitnehmerfreundliche Sozialpolitik, sondern auch politisch motivierte Restriktionen für die Wirtschaft: „Obwohl mit Ausnahme von jüdischem Vermögen das Prinzip des Privateigentums erhalten blieb, wurde die Verfügungsautorität der Unternehmer zunehmend eingeschränkt."4 Für die Banken galt dies beispielsweise in Bezug auf Devisenbewirtschaftung und Profite.

Die Banken galten in der nationalsozialistischen Ideologie von Beginn an nicht viel: So hatte der spätere Parteiideologe der NSDAP, Gottfried Feder, bereits 1919 sein „Manifest zur Brechung der Zinsknechtschaft“ veröffentlicht, 1919 den „Deutschen Kampfbund zur Brechung der Zinsknechtschaft“ gegründet und bis 1933 weitere einschlägige Schriften publiziert: „Die Juden“ und „Kampf gegen die Hochfinanz“. Ein weiterer Grund für die Schwächung der deutschen Banken lag in den Konsequenzen der Weltwirtschaftskrise, die 1931 durch den Zusammenbruch mehrerer deutscher Großbanken eine Bankenkrise herbeigeführt hatte $^{5}$, die Staatseingriffe provozierte. Sie verstärkten sich seit 1933 Zug um Zug,

${ }^{2}$ Henry A. Turner, Unternehmen unter dem Hakenkreuz, in: Lothar Gall/Manfred Pohl (Hrsg.), Unternehmen im Nationalsozialismus, München 1998, S. 15-36, hier S. 16 f.

${ }^{3}$ Vgl. Joachim Scholtyseck, Robert Bosch und der liberale Widerstand gegen Hitler 1933-1945, München 1999.

4 Turner, Unternehmen, in: Gall/Pohl (Hrsg.), Unternehmen im Nationalsozialismus, Zitat S. 19.

${ }^{5}$ Vgl. Horst Möller, Die Weimarer Republik. Eine unvollendete Demokratie, München ${ }^{10} 2012$, S. 259; Harold James, Deutschland in der Weltwirtschaftskrise 1924-1936, Stuttgart 1988, S. 275 ff. Aus der älteren Literatur vgl. die Gesamtdarstellung von Karl Erich Born, Die deut- 
wenngleich nun mit ideologischer Zielsetzung und gezielten Pressionen der NSDAP, beispielsweise auf die Personalpolitik der Banken.

Viele Banken hatten schon zu Beginn der NS-Herrschaft, manchmal in vorauseilendem Gehorsam, jüdische Vorstandsmitglieder und andere Juden in leitenden Positionen entlassen. Sie waren überdies einem wachsenden Druck von innen ausgesetzt, wenn eine große Zahl von Angestellten die nationalsozialistische Ideologie teilte und gegen den „Finanzkapitalismus“ oder jüdische Bankiers und Manager polemisierte. So wurde die Bayerische Vereinsbank (BV) als „Judenbank“ denunziert, weil sie mit der Berliner Mendelssohn Bank einen jüdischen Großaktionär hatte.

Die Konzentration der geschichtswissenschaftlichen Forschung auf wenige Großbanken hat dazu geführt, dass in der Regel über die Geschichte kleinerer oder mittlerer Institute wenig bekannt ist: Dies gilt für die Bayerische Hypotheken- und Wechsel-Bank (Hypo-Bank) und die Bayerische Vereinsbank, die Bayerische Staatsbank und andere Banken, für die entsprechende Forschungen erst noch zu leisten sind ${ }^{6}$. Diese Banken waren nicht überall im Reich präsent, sie spielten auch für die deutsche Besatzungspolitik keine Rolle. Neben den klassischen Sektoren des Bankgeschäfts, wie zum Beispiel die Geschäftsentwicklung, die mal stärker, mal weniger stark durch die politischen und wirtschaftlichen Rahmenbedingungen der nationalsozialistischen Diktatur bestimmt war, konzentrierten sich die für das Regime spezifischen Aktivitäten auf drei Sektoren: die Entlassung jüdischer Vorstandsmitglieder oder Mitarbeiter, die Beteiligung an „Arisierungen“ und das Verhalten gegenüber jüdischen Kapitaleignern.

Während der Begriff „Gleichschaltung“ für die Banken insofern eine begrenztere Bedeutung hatte, als sie keine Verfassungsorgane, staatliche Verwaltungen, politische Parteien oder gesellschaftliche Organisationen waren, trifft die Frage nach der möglichen „Resistenz“ den Kern: Banken waren kein „Hort des Widerstands“ oder der Opposition, ihre Vorstände bzw. Aufsichtsräte konnten - auch angesichts der ihnen gegenüber kritisch eingestellten Nationalsozialistischen Betriebszellen - allerdings den Versuch machen, sich der Einflussnahme von NSFunktionären und ihrer Ideologie zu entziehen.

Aus diesem Grund geht es in diesem Aufsatz im Kontext der Bankpolitik des NS-Regimes vor allem um folgende Fragen: Wie, wann und mit welchen konkreten

sche Bankenkrise, München 1967; ders., Geld und Banken im 19. und 20. Jahrhundert, Stuttgart 1977.

${ }^{6}$ Vgl. demnächst Möller, Regionalbanken im Dritten Reich; Eva Moser/Richard Winkler, Wegmarken. 125 Jahre Bankhaus H. Aufhäuser, München 1995; Angelika Baumann/Andreas Häusler (Hrsg.), München arisiert. Entrechtung und Enteignung der Juden in der NS-Zeit, München 2004; Wolfram Selig, „Arisierung“ in München. Die Vernichtung jüdischer Existenz 1937-1939, Berlin 2004; Geschichte des Finanzplatzes München. Mit Beiträgen von Markus A. Denzel, Albert Fischer, Rainer Gömmel, Margarete Wagner-Braun, Franz-Christoph Zeitler, hrsg. im Auftrag des Wissenschaftlichen Beirats des Instituts für bankhistorische Forschung e.V. von Hans Pohl, München 2007. Vgl. zuletzt Johannes Bähr/Axel Drecoll/Bernhard Gotto, Die Geschichte der Bayern LB, hrsg. vom Institut für Zeitgeschichte München-Berlin im Auftrag der Bayern LB, München/Zürich 2009. 
Zielen haben Bankenaufsicht und NS-Funktionäre versucht, die Autonomie der Bayerischen Vereinsbank einzuschränken und diese „gleichzuschalten“? Welchen Pressionen waren Vorstand und Aufsichtsrat in den einzelnen Phasen ausgesetzt und wie haben sie darauf reagiert? In welchem Maße gelang es dem Regime, in diesen Gremien nationalsozialistische Mitglieder durchzusetzen und wie haben sie dort agiert? Verlor die Bayerische Vereinsbank ihre Autonomie oder konnte sie sie wenigstens partiell bewahren?

\section{Bankpolitik im NS-Regime}

Überwachungsmaßnahmen in der Preispolitik, wie die Einsetzung eines Reichskommissars für Preisüberwachung am 15. Juli 1933 oder - speziell für die Banken von Interesse - das Gesetz über die Gewinnverteilung bei Kapitalgesellschaften (Anleihestockgesetz) vom 4. Dezember 1934, schienen auf die am «Gemeinwohl» orientierte Volksgemeinschaftsideologie zurückzugehen: Diesem Gesetz zufolge durfte der für ein Geschäftsjahr bar auszuschüttende Gewinn bei Kapitalgesellschaften - wie die Bayerische Vereinsbank eine war - sechs Prozent des eingezahlten Kapitals nicht überschreiten ${ }^{7}$. Mit dieser Regelung schuf das Reichswirtschaftsministerium „ein gesetzliches Instrument zur Lenkung langfristiger Anlagemittel in die industrielle Selbstfinanzierung. [...] Die Kapitalanlage in Aktien ermöglichte daher keine höhere Rendite als die Anlage in festverzinslichen staatlichen Wertpapieren, die mit $6 \%$ verzinst waren. “8

Einen wesentlichen Schritt zur Reglementierung der Banken bildete schließlich das Reichsgesetz über das Kreditwesen vom 5. Dezember 1934. Es war auf der Grundlage eines Entwurfs entstanden, den ein von der Reichsregierung Mitte 1933 eingesetzter „Untersuchungsausschuß für das Bankwesen“ im Dezember 1933 vorgelegt hatte und der kaum nationalsozialistisch geprägt war. Aufgabe dieser Enquetekommission war es, Präventivmaßnahmen vorzuschlagen, die künftig eine Bankenkrise wie die von 1931 verhindern sollten. Zwar folgte das Reichsgesetz über das Kreditwesen weitgehend dem Entwurf, doch wurde die Bankenaufsicht erheblich verschärft. Bei der Reichsbank entstand zu diesem Zweck eine eigene Behörde, das „Aufsichtsamt über das Kreditwesen“, das mit den schon vorher eingerichteten Reichskommissaren die Bankenaufsicht ausübte. Das Problem bestand hier nicht in dem insgesamt durchaus begründbaren (und auch nach 1945 geltenden) Prinzip der Bankenaufsicht, sondern in der Möglichkeit zu ihrer Ideologisierung im Sinne des Regimes. Christopher Kopper hat die Bankenpolitik während der 1930er Jahre „zwischen Marktwirtschaft und Dirigismus“ an-

\footnotetext{
${ }^{7}$ Vgl. Gesetz über die Gewinnverteilung bei Kapitalgesellschaften, §3, in: Ursachen und Folgen. Vom deutschen Zusammenbruch 1918 und 1945 bis zur staatlichen Neuordnung Deutschlands in der Gegenwart. Eine Urkunden- und Dokumentensammlung zur Zeitgeschichte, hrsg. von Herbert Michaelis und Ernst Schraepler unter Mitwirkung von Günter Scheel, 26 Bde, Berlin o.J., Bd. IX, S. 701-703, hier S. 701.

${ }^{8}$ Christopher Kopper, Zwischen Marktwirtschaft und Dirigismus. Bankenpolitik im „Dritten Reich“ 1933-1939, Bonn 1995, Zitat S.151.
} 
gesiedelt ${ }^{9}$ - eine Charakterisierung, die insgesamt für die Privatwirtschaft im NSRegime zutrifft.

Eine Reihe weiterer gesetzlicher Vorgaben ließ nicht lange auf sich warten. Beispielsweise wurden aufgrund eines Vorschlags des Reichswirtschaftsministeriums vom 24. Januar 1935 die Zinsen von Pfandbriefen herabgesetzt, so dass bei allen nichtstaatlichen Schuldverschreibungen der Zinssatz auf 4,5 Prozent gesenkt wurde. Am 8. Februar 1935 folgte das Gesetz über die Zinsermäßigung bei öffentlichen Anleihen: Die erhebliche Zinssenkung am Kapitalmarkt sollte eine Zinssenkung am Geldmarkt vorbereiten und damit die Voraussetzungen einer Kreditpolitik des „billigen Geldes“ schaffen ${ }^{10}$.

Die völlige Indienstnahme der Wirtschaft einschließlich der Banken für die politischen Ziele des NS-Regimes zeigte sich auch in Hitlers Begründung für den Vierjahresplan: „So wie die politische Bewegung in unserem Volk nur ein Ziel kennt, die Lebensbehauptung unseres Volkes und Reiches zu ermöglichen, d. h. alle geistigen und sonstigen Voraussetzungen für die Selbstbehauptung unseres Volkes sicherzustellen, so hat auch die Wirtschaft nur diesen einen Zweck. Das Volk lebt nicht für die Wirtschaft oder für die Wirtschaftsführer, Wirtschafts- oder Finanz-Theorien, sondern die Finanz und die Wirtschaft, die Wirtschaftsführer und alle Theorien haben ausschließlich diesem Selbstbehauptungskampf unseres Volkes zu dienen." ${ }^{11}$

In diesem Text werden Hitlers Ressentiments gegen die Finanzwelt spürbar, vor allem aber lieferte er die Begründung für zahlreiche weitere Eingriffe, so zum Beispiel für das Gesetz gegen Wirtschaftssabotage vom 1. Dezember $1936^{12}$, mit dem die Verbringung von Vermögen ins Ausland unter Strafe gestellt, ja sogar mit der Todesstrafe bedroht wurde: Dieses Gesetz richtete sich nicht nur, aber in erster Linie gegen Emigrationswillige, also vor allem gegen deutsche Juden, die nach der ständigen Verschärfung antijüdischer Aktionen - von den 1933 beginnenden Entrechtungen über die Nürnberger Gesetze von 1935 bis zu den Pogromen vom 9. November 1938 sowie der sogenannten Arisierung - Deutschland verlassen mussten.

Auch die erste der beiden Gleichschaltungsaktionen des NS-Regimes gegen die Banken, die 1938 und 1942/43 erfolgten, besaß nicht zuletzt eine antijüdische Stoßrichtung; sie wirkte sich, wie noch gezeigt wird, in besonderem Maße auf die Bayerische Vereinsbank aus, bevor seit 1942 die Kritik der NSDAP an ver-

\footnotetext{
${ }^{9}$ Vgl. ebenda; vgl. auch Jens Jessen (Hrsg.), Reichsgesetz über das Kreditwesen vom 5. Dezember 1934 mit Begleittext, Erläuterungen und Begründung, Berlin 1934.

${ }^{10}$ Kopper, Zwischen Marktwirtschaft und Dirigismus, S. 157.

${ }^{11}$ Hitlers Denkschrift zum Vierjahresplan 1936, eingeleitet und kommentiert von Wilhelm Treue, in: VfZ 3 (1955), Dokument S. 204-210, Zitat S. 206. Es existierten seinerzeit lediglich drei Exemplare, sie waren damals nur Hermann Göring und dem Reichskriegsminister und Oberbefehlshaber der Wehrmacht Werner von Blomberg bekannt, da die Denkschrift auch die kriegspolitischen Ziele Hitlers ohne Umschweife beim Namen nannte.

${ }^{12}$ Vgl. Textauszug in: Ursachen und Folgen, Bd. X, S. 557.
} 
meintlich übertriebenen Bankgewinnen unter Kriegsbedingungen eine regelrechte „Offensive der Partei gegen die Banken“13 einleitete.

\section{Die Bayerische Vereinsbank nach der Weltwirtschaftskrise}

Die 1869 gegründete Bayerische Vereinsbank war eine Bank mit differenzierten Geschäftssparten im Hypotheken- und im Bankgeschäft. Dazu gehörten lang- und kurzfristige Kredite, Einlagen, Depots und bald auch das Emissionsgeschäft. 1899 trat das Hypothekenbankgesetz in Kraft, wodurch die Bayerische Vereinsbank, die bereits nach ihrer Satzung von 1869 das Kredit- und das Hypothekengeschäft parallel betreiben durfte, den Rechtsstatus einer „Gemischten Bank“ erhielt. In den ersten Jahrzehnten blieb sie auf München beschränkt, gründete dann aber bis 1913, zum Teil durch Übernahmen von Privatbanken, insgesamt zwölf Filialen und zwei Wechselstuben innerhalb des rechtsrheinischen Bayern. Als gemischte bayerische Regionalbank ähnelten Struktur und Geschäftsmodell der Bayerischen Hypotheken- und Wechsel-Bank. 1920/21 übernahm die Bayerische Vereinsbank das Bankgeschäft der Bayerischen Handelsbank sowie der Vereinsbank in Nürnberg, die später als reine Hypothekenbanken hundertprozentige Tochterunternehmen der BV wurden.

Größere praktische Bedeutung hatte die 1920/21 begründete Kooperation, das sogenannte Freundschaftsverhältnis mit der Berliner Mendelssohn-Bank, die insgesamt 16 Prozent des auf 375 Millionen Mark erhöhten Stammkapitals der BV übernahm. Die Zahl der BV-Filialen wechselte, Ende 1923 waren es insgesamt 54. Das zweimal aufgestockte Aktienkapital betrug nominal zwischen 1933 und 1945 31,05 Millionen RM. Während das Hypothekenbankgeschäft schließlich reichsweit war, beschränkte sich das Bankgeschäft auf Bayern. Im Jahr 1933 betrug die Bilanzsumme 630,5 Millionen RM bei einem Reingewinn von 2,024 Millionen und einer Dividende von sechs Prozent. Die Zahl der Mitarbeiter lag im Jahr 1933 bei 1.527. Die BV war damit nach der Bayerischen Hypotheken- und WechselBank die wirtschaftlich bedeutendste gemischte Regionalbank in Bayern.

Als der Vorstand der Bayerischen Vereinsbank am 12. April 1932 seinen Geschäftsbericht für das Krisenjahr 1931 vorlegte, beklagte er nach einer Analyse der weltwirtschaftlichen Entwicklung die Differenz ökonomischen und politischen Denkens: „Es fehlt das Bewußtsein einer Verbundenheit der gesamten Weltproduktion, einer Verflechtung des Welthandels, es fehlt die ausgleichende Wirkung der Weltmärkte, auf die schließlich die ganze Welt angewiesen ist. Die Erkenntnis dieser Situation ist in der Wirtschaft der ganzen Welt vorhanden, aber die Politik aller Staaten geht andere Wege als die, die zu einer Abhilfe notwendig wären. "14 Dieses Urteil war nur zu gerechtfertigt, doch sollte sich die Situation durch die zunehmende Destabilisierung der europäischen Demokratien keines-

\footnotetext{
${ }^{13}$ Harold James, Die Rolle der Banken im Nationalsozialismus, in: Gall/Pohl (Hrsg.), Unternehmen im Nationalsozialismus, S. 25-36, hier S. 33.

${ }^{14}$ Hierzu und zum Folgenden siehe Geschäftsbericht für das Geschäftsjahr 1931, S. 4, in: Historisches Archiv der UniCredit Bank AG München, D-BV-KOM-PUB-63.
} 
wegs verbessern, sondern weiter verschlechtern, zumal in Deutschland: Die seit 1933 verfolgte Autarkiepolitik des NS-Regimes war das genaue Gegenteil der hier begründeten Notwendigkeit einer „ausgleichenden Wirkung der Weltmärkte“. Doch wies schon der Interventionismus der Regierung Brüning in die kritisierte Richtung. Ein Beispiel war die Regelung der Devisenbewirtschaftung von 1931, die durch die spätere „Reichsfluchtsteuer“ verschärft wurde.

Der Vorstand der Vereinsbank beklagte zwar das durch Notverordnungen und staatliche Hilfen verursachte „Eindringen des Staatskapitalismus in das private Bankgewerbe“, doch sah er die Ursache für diese Entwicklung in der Bankenkrise von 1931. Insgesamt vertrat der Vorstand die Meinung, die Zwangskonvertierung von Pfandbriefen und Anleihen schädigte ebenso wie das „eiserne Kleid für die Zinsen“ den Kapitalmarkt. Die Schaffung einer Reichsaufsicht für das Bankgewerbe wurde als populistisches Zugeständnis an die öffentliche Meinung gewertet, von ihrer Handhabung werde es abhängen, wie sie sich künftig auswirke: „Es ist der Mut der Regierung zu bewundern, daß sie die Verantwortung für eine obrigkeitliche Beeinflussung der Bankenpolitik auf sich nehmen will, obwohl gerade in der jetzigen Krise trotz aller Staatsaufsicht bei verschiedenen öffentlich-rechtlichen Bankinstituten gleiche Schwierigkeiten, wie sie im privaten Bankgewerbe auftraten, nicht zu verhindern waren."

In dieser Diagnose der Finanzpolitik der frühen 1930er Jahre zeigt sich ein weiteres Mal, warum der fundamentale Bruch des Jahres 1933 in Hinblick auf die Bankpolitik nicht in seiner Tragweite erkannt wurde: Die zunehmende Reglementierung und „obrigkeitliche Beeinflussung“ begann bereits vor der nationalsozialistischen Revolution, das Hineingleiten aus entparlamentarisierten, präsidialautoritären Herrschaftsstrukturen in diktatorische ließ den Bruch des politischen Systems fälschlich als Modifikation und nicht als Zäsur erscheinen, die Frage nach wirtschaftlichem Aufschwung und Besserung dominierte alles. Andererseits zeigen gerade die liberalen ökonomischen und handelspolitischen Grundprinzipien, die die weltwirtschaftliche Analyse des BV-Vorstands leiteten, wie unvereinbar die wirtschaftspolitische Konzeption des Nationalsozialismus und die der Banken tatsächlich war, setzten Letztere doch ohne Wenn und Aber auf ein freies Bankgewerbe und Unternehmertum, auf private Initiative anstelle kollektivistischer Strukturen.

Wie andere Regionalbanken überstand auch die Bayerische Vereinsbank die Wirtschaftskrise vergleichsweise gut. Als Regionalbank mit starkem Engagement im Hypothekenbankgeschäft für die Landwirtschaft war sie weniger direkt als die nationalen Großbanken, die einen globaleren Aktionsradius besaßen und in der Großindustrie aktiv wurden, in den Strudel der Weltwirtschaftskrise geraten. Insofern konnte der Vorstand berichten: „Gemessen an den Ereignissen des Berichtsjahres und dem gegenwärtigen Zustande des deutschen Bankgewerbes, kann unser Institut mit seiner Stellung zufrieden sein. Da wir Auslandsgelder, trotz mannigfacher Anregungen, niemals in einem nennenswerten Ausmaße he- 
reingenommen hatten, wurden wir von dem Abzuge solcher Gelder so gut wie nicht betroffen." 15

\section{Geschäftsentwicklung der Bayerischen Vereinsbank und die poli- tische Lage}

Überblickt man die Geschäftsentwicklung der Bayerischen Vereinsbank während der nationalsozialistischen Diktatur, dann ist zunächst festzustellen, dass ihre Aktivitäten sich weiterhin zum größten Teil auf Bayern beschränkten. Außer in den beiden Filialen im Sudetenland - die sich aber im Wesentlichen mit den üblichen Bankgeschäften einer deutschstämmigen Klientel befasst haben dürften - machte das Institut also keinerlei Bankgeschäfte in den von Deutschland besetzten Staaten. An „Arisierungen“ oder später an Kriegsgeschäften im Ausland war die Bayerische Vereinsbank nicht beteiligt. Die Gewinne der Bank steigerten sich mit der Konjunkturentwicklung seit der zweiten Hälfte der 1930er Jahre, erreichten aber erst um 1937 den Stand vor der Bankenkrise von 1931. Eine deutliche Vermehrung der Aktivitäten und auch der Gewinne ergab sich in den ersten Kriegsjahren, bevor letztere 1943 wieder ungefähr auf den Stand zurückfielen, den sie vor der wirtschaftlichen Erholung erlangt hatten. Die zeitweilige Erhöhung der Überschüsse war aber nur in begrenztem Maße eine reale Steigerung, sondern zum erheblichen Teil auf die inflatorische Entwicklung zurückzuführen.

Die Steigerung fiel insgesamt in der Hypothekenabteilung erheblich geringer aus als in der Bankabteilung, deren Volumen seit 1941 auch aufgrund der Inflation in die Höhe schoss und das der Hypothekenabteilung bei weitem überstieg ${ }^{16}$. Das Hypothekengeschäft, konzentriert auf kleinere und mittlere Hypotheken, erholte sich zwar in der zweiten Hälfte der 1930er Jahre, doch vergleichsweise langsam und verminderte sich bereits wieder auf dem Höhepunkt des Krieges. Die Entwicklung von Bank- und Hypothekengeschäft war in diesen Jahren des öfteren gegenläufig, wodurch die eine Abteilung das mäßige Geschäft der anderen kompensieren konnte. Das vom Hypothekengeschäft abhängige Pfandbriefgeschäft war seit Beginn der Wirtschaftskrise am Ende der Weimarer Republik mit Restriktionen belegt, die Emissionssperre wurde zwar seit 1940 etwas gelockert, blieb aber weiterhin hinderlich, zumal die Nachfrage enorm war. In Deutschland nahm zwischen 1932 und 1938 der Umlauf an Pfandbriefen und Kommunalobligationen um 422 Mio. RM ab, die Emission öffentlicher Anleihen verfünffachte sich im Gegensatz zu dieser Entwicklung im gleichen Zeitraum von 3,5 Mrd. auf 17,7 Mrd. „Pfandbriefe und Kommunalobligationen dienen der Investition für den Frieden; in der Periode der Aufrüstung, aber auch schon vorher, während der zentral gesteuerten Arbeitsbeschaffung, war kein Platz für sie." 17 Auch hieraus er-

15 Geschäftsbericht für das Geschäftsjahr 1931, S. 7 f., in: Historisches Archiv der UniCredit Bank AG München, D-BV-KOM-PUB-63.

${ }^{16}$ Vgl. Franz Steffan, Die Bayerische Vereinsbank 1869-1969. Eine Regionalbank im Wandel eines Jahrhunderts, München 1969, Schaubild 68 u. S. 292.

17 Ebenda, S. 292. 
gibt sich die prinzipielle Interessendifferenz der staatlich gelenkten Wirtschaft und der für die Banken charakteristischen liberalen Marktorientierung: Jenseits der ideologischen oder konzeptionellen Gegensätzlichkeit kommt der in den Geschäftsberichten - trotz drohender Repressionen - immer wieder geäußerten Kritik besondere Bedeutung für das Verhältnis der Bank zum nationalsozialistischen Regime zu.

Auf nachhaltige Weise wurden die Aktivitäten der Bayerischen Vereinsbank seit Ende der 1930er Jahre durch das Privatkundengeschäft belebt. Im Krieg wuchs das Bedürfnis, für Notzeiten etwas auf die hohe Kante zu legen, außerdem verminderte sich das Warenangebot, während die Kaufkraft stieg: Dies führte zu einer außergewöhnlichen Erhöhung, ja nahezu einer Verdoppelung der Kontenzahl bei ungefähr gleichbleibender Zahl der Filialen sowie massenhaften kurzfristigen Geld- und Spareinlagen, darunter der Anlage von Wertpapierdepots. Insgesamt aber hat die Bayerische Vereinsbank während der Diktatur keine im Vergleich zur vorherigen Entwicklung außergewöhnlichen Privatkunden-Geschäfte gemacht. Besser verlief hingegen das Firmenkundengeschäft. Da sich die Rüstungsproduktion während des Zweiten Weltkriegs zum wichtigsten Industriezweig entwickelte, wurden Rüstungsbetriebe naturgemäß zu den größten Kreditnehmern der Banken. Mit Abstand größter Kreditkunde der Bayerischen Vereinsbank war bis Kriegsende das Unternehmen des Flugzeugkonstrukteurs Willy Messerschmitt in Augsburg ${ }^{18}$, dessen Sanierung durch das Bankhaus Seiler \& Co (ehemals H. Aufhäuser) und den Finanzmakler Münemann mithilfe von Schuldscheindarlehen nicht ausreichte. Messerschmitt, der von 1940 bis 1945 dem Aufsichtsrat der Bayerischen Vereinsbank angehörte, stellte unter anderem das im Zweiten Weltkrieg eingesetzte Jagdflugzeug Me 109 sowie das erste in Serie produzierte Düsenjagdflugzeug Me 262 her, die er konstruiert hatte.

Indirekte Profite aus den Krediten für die Rüstungsindustrie fielen bei der Bayerischen Vereinsbank nicht so entscheidend ins Gewicht, dass sie den Gewinn in die Höhe getrieben hätten. Die Bayerische Vereinsbank konnte zwischen 1924 und 1945 im Vergleich zu den Jahren vor dem Ersten Weltkrieg insgesamt „nur mäßige Erträge erzielen“19, obwohl sie in Konkurrenz zu anderen ähnlichen Banken nicht schlecht dastand. Die Einnahmen des schlechtesten Jahres 1932 waren um 30 Prozent niedriger als im besten Jahr dieses Zeitraums 1942. Der Rohertrag und noch weniger der Reingewinn hielten mit dem Wachstum des Volumens „bei weitem nicht Schritt, die natürliche Folge davon, daß eine gewinnbringende Anlage der Geldfülle mit normalen Zinsspannen nicht möglich war und die Erträge aus dem Effekten- und Konsortialgeschäft bei weitem nicht mehr die Rolle spielten wie früher". Einen ähnlichen Rückgang erlebten teilweise aufgrund des Vordringens des Wechselgeschäfts die Provisionen ${ }^{20}$.

\footnotetext{
18 Vgl. ebenda, S. 291.

${ }^{19}$ Ebenda, S. 294.

${ }^{20}$ Ebenda, S. 295 (Zitat) u. S. 273.
} 


\section{Die Bayerische Vereinsbank und die NS-Ideologie}

Veränderte sich der Stil der Geschäftsberichte, nachdem die nationalsozialistische Diktatur sich etabliert und zum größeren Teil normativ, zu einem erheblichen Teil aber auch faktisch die Gleichschaltung von Staat, Gesellschaft, Wirtschaft und Kultur vollzogen hatte? Welche Antwort gibt der am 11. April 1935 vorgelegte Geschäftsbericht für das Jahr 1934 auf diese Frage? Die Antwort lautet: Der Vorstand der Bayerischen Vereinsbank passte seinen Stil gar nicht, den Inhalt nur vereinzelt und völlig sachbezogen an die neuen Verhältnisse an. Tatsächlich blieben die Berichte der Bayerischen Vereinsbank - außer in vereinzelten Bemerkungen - frei von ideologiegetränkten Äußerungen. Dies war zwar auch bei anderen Banken die Regel, doch dominierte in Vorstand und Aufsichtsrat der Bayerischen Vereinsbank zumindest bis 1938 eine erkennbare Distanz zum NS-Regime. Der Geschäftsbericht der Bank wies auf die Besserung der Wirtschaftslage und damit die Senkung der Erwerbslosigkeit in Deutschland hin, doch alles in einer ausgesprochen nüchternen Diktion. Dafür charakteristisch sind bereits die einleitenden Sätze: „Während des Berichtsjahres 1934 stand die deutsche Wirtschaft weiterhin in erster Linie unter der Einwirkung der umfassenden und vielfältigen wirtschaftspolitischen Maßnahmen des Staates. Die verschiedenen Arbeitsbeschaffungsprogramme des Reiches und der Länder und die sonstigen zahlreichen Staatsaufträge beeinflussten ausschlaggebend die Konjunktur und den Aufschwung der Wirtschaft." 21

Viele staatliche Maßnahmen wurden jedoch als Versuche charakterisiert, bestimmte wirtschaftspolitische Ziele zu erreichen, ohne dass dies immer voll gelungen sei. Der Vorstand verwendete Begriffe wie „Überwachung und Reglementierung“ und wenn er sie mit der Bewertung "in dieser Situation notwendige Maßnahmen“ etikettiert, folgte in der Regel der Hinweis auf die negativen Begleiterscheinungen solcher staatlichen Vorschriften. Nachdrückliche Mahnungen schlossen sich an: Die Reichsregierung habe doch wiederholt betont, „grundsätzlich an der Freiheit der Wirtschaft und der Förderung der Initiative der einzelnen wirtschaftenden Personen festhalten“ zu wollen, da sie diese „als wesentliche Triebkräfte der wirtschaftlichen Entwicklung“ ansehe. Die Bank machte mit solchen Formulierungen immer wieder den Versuch, die nationalsozialistische Regierung beim Wort zu nehmen. Sie bemühte sich dabei offensichtlich um die verfassungsrechtliche Unterscheidung von Partei und Staat, um der ideologischen und machtpolitischen Deformierung administrativer Regelungen mit Sachargumenten entgegenzuwirken. Überdies handelte es sich um die regelmäßige Wiederholung des Appells, der Staat möge eine freie Wirtschaft ermöglichen, weil nur sie die Krise meistern könne. Auch hier fand sich der mit Nachdruck vorgetragene Hinweis, die Gesundung der Binnenkonjunktur reiche keineswegs aus, es müsse „mit allen Mitteln auf die Vermehrung der deutschen Ausfuhr“ hingearbei-

${ }^{21}$ Hierzu und zum Folgenden siehe Geschäftsbericht für das Geschäftsjahr 1934, S. 4, in: Historisches Archiv der UniCredit Bank AG München, D-BV-KOM-PUB-66. 
tet werden: Dies sei in Zukunft die wichtigste Aufgabe freier Unternehmerinitiative.

Das Gesetz zur Ordnung der nationalen Arbeit vom 20. Januar 1934 kommentierte der Vorstand der Bayerischen Vereinsbank mit den Worten, es sei in der Wirtschaft „aufrichtig begrüßt“ worden. Der Kommentar fiel jedoch in fast akrobatischen Formulierungen denkbar allgemein aus: „Die hohen Gedanken der Verbundenheit aller Betriebsangehörigen, des gegenseitigen Vertrauens und der Kameradschaft sind geeignet, den Frieden der gemeinsamen Arbeit zu sichern, die Grenzen des Wünschenswerten und des Möglichen in den sozialen Fragen nahe aneinander zu rücken." Und das Reichsgesetz über das Kreditwesen vom 5. Dezember 1934 dokumentiere in den Augen des BV-Vorstands „durch die Fülle der den Banken auferlegten Pflichten und Kontrollen die Bedeutung des Bankgewerbes“. Im Übrigen müsse man aber die Handhabung durch die amtlichen Stellen abwarten, erst dann könne beurteilt werden, ob es dem Bankgewerbe gelinge, „das schwere Problem einer gesicherten Ertragsfähigkeit zu lösen“22.

Auch die Geschäftsberichte der späteren Jahre, die der Vorstand jährlich für die Hauptversammlung erstattete, blieben von nationalsozialistischer Ideologie weitgehend frei. Bis 1938 finden sich nur gelegentlich NS-affine Aussagen, ab 1941 gar nicht mehr: Lediglich die im Frühjahr 1940 für 1939 und im Frühjahr 1941 für 1940 erstatteten Berichte enthalten eine positive Bewertung der „Blitzsiege“ und ihrer Folgen, einmal auch eine Art Treuebekenntnis zum „Führer“. Die positiven Bemerkungen über den Anschluss Österreichs und des Sudetenlandes sind nicht zwangsläufig als nationalsozialistisch zu werten: Diese Aktionen stießen wegen des nach 1919 in beiden Ländern beabsichtigten, aber in den Friedensverträgen in Versailles und Saint Germain untersagten Zusammenschlusses sowie der drei Millionen deutschstämmigen Sudetendeutschen auch außerhalb der NSDAP auf breite Zustimmung. Die wirtschafts- und finanzpolitischen Maßnahmen des Regimes fanden zwar teilweise Zustimmung, regelmäßig aber auch vorsichtige Kritik: Der Vorstand argumentierte etwa rein ökonomisch bzw. bankfachlich gegen zu starke staatliche Reglementierung sowie die Autarkiepolitik, selbst wenn solche Kritik zuweilen etwas verklausuliert formuliert wurde.

Die gewissen Unterschiede der Berichte im Hinblick auf den Nationalsozialismus in den einzelnen Zeitphasen sind vermutlich auf die erwähnten Gleichschaltungsmaßnahmen des Regimes von 1938 zurückzuführen, die zumindest zu gröBerer Vorsicht zwangen. Die Ausblendung des politischen Kontextes ab 1942 ist aber so nicht zu erklären: Da das NS-Regime damals erneut Pressionen auf die Bayerische Vereinsbank ausübte, hätten verbale Zugeständnisse in ihren Berichten näher gelegen. Da der ideologische Druck sich kaum vermindert hatte, spielte möglicherweise eine Desillusionierung selbst bei solchen Angehörigen der Bank eine Rolle, die der NSDAP nahe standen oder ihr angehörten. Zu unterscheiden von dieser sachlichen und zeitlichen Differenzierung sind allerdings die zahl-

\footnotetext{
${ }^{22}$ Geschäftsbericht für das Geschäftsjahr 1934, S. 6, in: Historisches Archiv der UniCredit Bank AG München, D-BV-KOM-PUB-66.
} 
reichen eindeutig nationalsozialistischen Äußerungen, die von der Nationalsozialistischen Betriebszellenorganisation (NSBO) in der Belegschaft kamen.

Vergleicht man die Geschäftsberichte des Vorstands mit der Betriebszeitschrift „Betrieb und Vertrauen“ und den Erklärungen des Betriebsobmanns Emil Kronawitter, wird der Kontrast augenfällig. Dazu trugen nicht nur regelmäßige Bezugnahmen der NSBO auf Hitler-Reden oder den Vorsitzenden der Deutschen Arbeitsfront (DAF), Dr. Robert Ley, bei, sondern auch inhaltliche Bekenntnisse zu Kernelementen der NS-Ideologie, beispielsweise zur „,arischen Abstammung“; die „kameradschaftliche Verbundenheit“ im Betrieb wurde zunehmend und verstärkt seit den antijüdischen Nürnberger Gesetzen von 1935 rassistisch definiert ${ }^{23}$. Emil Kronawitter, der als Betriebsobmann zugleich stellvertretender Betriebsführer und stellvertretendes Vorstandsmitglied war, erhielt dennoch durch die Betriebsobleute der Bayerischen Vereinsbank 1946 eine etwas überraschende Entlastung: In diesem „Persilschein“ hieß es, seine „innerlich widerstrebende Einstellung zu den Absichten der DAF und der Parteifunktionäre war nicht zu verkennen. Die im Betrieb vorhandenen sehr zahlreichen überzeugten Nazigegner hatten stets das sichere Gefühl, dass Herr Direktor Kronawitter, wo immer er nur konnte, in milderndem Sinne seinen Einfluss geltend zu machen suchte. Sein spät erfolgter Beitritt zur Partei wurde als reine Formsache angesehen und war nicht dazu ange-

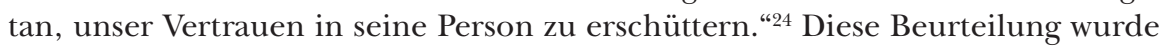
vom Betriebsrat im Spruchkammerverfahren gegen Kronawitter am 12. Dezember 1947 bestätigt.

Seit dem 1. Mai 1937 besaß die Bayerische Vereinsbank auch eine „Betriebsordnung“, deren Phraseologie den Formeln der Werkzeitschrift ähnelte, wenngleich sie im Wesentlichen die ursprüngliche, bis dahin gültige nicht nationalsozialistisch formulierte Dienstanweisung fortführte. Aufgrund des Gesetzes zur Ordnung der nationalen Arbeit wurde ein Vertrauensrat gebildet, der den Betriebsrat ablöste. Bei der Neukonstruktion entfiel die Arbeitnehmervertretung im Aufsichtsrat, in dem künftig nur Aktionärsvertreter saßen.

Die Leitung der Bayerischen Vereinsbank reagierte in ihren Geschäftsberichten wohl nicht allein wegen neuer Regelungen, sondern zur Beeinflussung der Belegschaft mit ausführlichen Sozialberichten. Sie stellte das - von jeher verfolgte, aber nicht in dieser offensiven Weise präsentierte - sozialpolitische Engagement der Bayerischen Vereinsbank heraus, dabei sind ideologische Anklänge nur vereinzelt und in sparsamer Form spürbar. Der sozialstaatlichen Komponente des NS-Regimes sollte offenbar - wie es z. B. für das Berichtsjahr 1941 erkennbar ist Rechnung getragen werden: „Wir glauben unsere stets ernst genommenen Fürsorgepflichten auch im Berichtsjahre wieder voll erfüllt zu haben. “25

${ }^{23}$ E. Kronawitter, Betriebsführung, Betriebsgemeinschaft und Soziales, in: Betrieb und Vertrauen, 3. Jg., Folge 7, S. 121, in: Historisches Archiv der UniCredit Bank AG München, DBV-KOM-PUB-966.

${ }^{24}$ Erklärung der Betriebsobleute der Bayerischen Vereinsbank vom 8. 2. 1946, in: Historisches Archiv der UniCredit Bank AG München, D-BV-Per-A-1318.

${ }^{25}$ Geschäftsbericht für das Geschäftsjahr 1941, S. 13, in: Historisches Archiv der UniCredit Bank AG München, D-BV-KOM-PUB-73. 


\section{Kontinuität und Wechsel in Vorstand und Aufsichtsrat}

Bis 1938 besaßen die beiden Großaktionäre der Bayerischen Vereinsbank, die von Paul Reusch geleitete Gutehoffnungshütte und MAN sowie die MendelssohnBank, eine starke Stellung, die zugleich die Kontinuität zu den Jahren der Weimarer Republik verkörperte. Nun aber verschärfte das NS-Regime erneut die antijüdische Politik, wodurch die Bayerische Vereinsbank unmittelbar betroffen wurde: Das Jahr 1938 ist deshalb als Jahr der „Gleichschaltung“ der Vereinsbank bezeichnet worden ${ }^{26}$. Das Regime versuchte damals, stärkeren Einfluss auf die Leitung der Bank, insbesondere auf die Besetzung von Vorstand und Aufsichtsrat zu gewinnen. Es handelte sich in diesem Fall um zwei parallele, jedoch ineinandergreifende Vorgänge, einen erzwungenen Eignerwechsel bei den Großaktionären und eine personalpolitische Nötigung.

Allerdings kann trotz der 1938 erfolgenden generellen Verstärkung des nationalsozialistischen Einflusses auf das Bankwesen im Falle der Bayerischen Vereinsbank wohl kaum von einer „Gleichschaltung“ im engeren Sinn gesprochen werden: Tatsächlich handelte es sich um eine begrenzte Anpassung an die realen Machtverhältnisse, die vom politischen und ökonomischen Umfeld der Bank und von der Bankenaufsicht des Reiches bestimmt wurden. Die Reichskommissare wirkten nicht zwangsläufig als „Gleichschaltungskommissare“, da ihre Funktion vor 1933 nicht politisch definiert war. Reichskommissare waren für die Bayerische Vereinsbank wie für andere Hypothekenbanken schon in den 1920er Jahren eingesetzt worden, was seinen Grund in der Staatsaufsicht nach dem Hypothekenbankgesetz von 1899 hatte. Zwar war ihre Stellung schon seit der Bankenkrise von 1931 verstärkt worden, doch steigerte dies nach 1933 nicht zwangsläufig den nationalsozialistischen Einfluss auf die jeweilige Bank, vielmehr hing dessen Ausmaß von der Person des Kommissars ab. Im Falle der bayerischen Banken nahm diese Funktion meist ein Beamter des Bayerischen Staatsministeriums für Wirtschaft wahr, seit dem 2. April 1931 Ministerialrat Dr. Leonhard Meukel, der im Prinzip eher auf der Seite der Bank als auf der der NSDAP stand und sich offenbar nicht zur politischen Überwachung der Bayerischen Vereinsbank berufen fühlte. Nach seinem Tod 1944 bescheinigte ihm der Vorstand, die Bank in schwierigen Situationen stets unterstützt zu haben ${ }^{27}$.

Anders lagen die Dinge mit Blick auf Regierung und Partei. NSDAP-Funktionäre bzw. von diesen beherrschte Organisationen und das Reichswirtschaftsministerium versuchten von Beginn an, wie bei anderen Regionalbanken, auch in der Bayerischen Vereinsbank ihren Einfluss zu verstärken. Dabei ermöglichte die Satzung der Vereinsbank unauffällig die Ausübung äußeren Drucks: Gemäß $§ 10$ musste in jedem zweiten Jahr mit der ordentlichen Hauptversammlung die Hälfte der Mitglieder des Aufsichtsrats in der Reihenfolge ihrer Amtsdauer ausscheiden,

\footnotetext{
${ }^{26}$ Heinrich VII. Reuss, Bayerische Vereinsbank, unveröff. Dokumentation, in: Historisches Archiv der UniCredit Bank AG München, D-BV-KOM-A-1585.

${ }^{27}$ Geschäftsbericht für das Geschäftsjahr 1944 (August 1945), in: Historisches Archiv der UniCredit Bank AG München, D-BV-LO-A-1538
} 
gegebenenfalls durch Auslosung. 1933/1934 gehörten dem Aufsichtsrat 18 Mitglieder, dem Vorstand 4 sowie 2 stellvertretende Mitglieder an. Regelmäßig anwesend war bei den Sitzungen beider Gremien Reichskommissar Dr. Meukel, zugleich Senatspräsident am Oberlandesgericht München. An der Größe der Gremien änderte sich danach nichts, wohl aber an der Zusammensetzung, wobei trotz turnusmäßigem Wechsel die Kontinuität beachtlich blieb, da - soweit dies möglich war - meist Wiederwahl erfolgte. Selbst nach den mehrfachen massiven personellen Einflussnahmen des NS-Regimes waren zehn Jahre nach der NSMachtergreifung im März 1943 noch Mitglieder aus der Weimarer Zeit in den Führungsgremien vertreten: Im Aufsichtsrat waren dies unter anderen der Vorsitzende, Bankdirektor a. D. August Bauch, der Münchner Rechtsanwalt Hanns Dahn, der Bankdirektor a. D. Ludwig Hammon, der Münchner Unternehmer Franz Kustermann sowie im Vorstand Karl Butzengeiger und Dr. Wilhelm Biber.

Der massive personelle Gleichschaltungsversuch von NSDAP-Funktionären hatte zwar quantitativ begrenzten Erfolg, doch gelang es ihnen, einige zentrale Persönlichkeiten aus dem Aufsichtsrat und dem Vorstand zu vertreiben. Das galt vor allem für den Aufsichtsratsvorsitzenden Paul Reusch, der innerhalb der Bank Garant für Kontinuität war und der im Verein mit seinem Vorgänger Fritz Neumeyer den nationalsozialistischen Zugriff auf die Bank jahrelang blockierte. Am 29. März 1938 erklärte er seinen Austritt aus dem Aufsichtsrat ${ }^{28}$. Aber damit nicht genug: Das große, von Paul Reusch vertretene Aktienpaket der Gutehoffnungshütte sollte auf Umwegen an die NSDAP verkauft werden, ohne dass es der Partei allerdings gelang, dafür einen eigenen Repräsentanten in den Aufsichtsrat zu entsenden.

Paul Reusch ${ }^{29}$ war ein dominanter Charakter, unangepasst, selbstbewusst und dickköpfig, eine große Unternehmerpersönlichkeit. In der Weimarer Republik besaßen die wirtschaftlichen Interessen der Großindustrie für ihn eindeutig Priorität, ihnen wollte er die Politik dienstbar machen. Er stand zunächst auf dem äußersten rechten Flügel der Deutschen Volkspartei und agierte dann zunehmend antidemokratisch. Dennoch war er weder Nationalsozialist noch Antisemit. Von Beruf Bau- und Hütteningenieur, wurde der 1868 geborene Reusch 1905 Vorstandsmitglied und 1909 Vorstandsvorsitzender bzw. später Generaldirektor der Gutehoffnungshütte AG in Oberhausen, deren Mehrheitseigner die Familie Haniel war. Unter seiner Leitung expandierte das Unternehmen stark, es erwarb zahlreiche Beteiligungen, unter anderem 1921 die Aktienmehrheit bei der MAN (Maschinenfabrik Augsburg und Nürnberg). Um die öffentliche Meinung beeinflussen zu können, kaufte er für das Unternehmen drei süddeutsche Zeitungen,

${ }^{28}$ Geschäftsbericht für das Geschäftsjahr 1938, S. 15, in: Historisches Archiv der UniCredit Bank AG München, D-BV-KOM-PUB-70.

${ }^{29}$ Vgl. die Biografie von Peter Langer, Macht und Verantwortung. Der Ruhrbaron Paul Reusch, Essen 2012, die allerdings nicht auf Quellen der Bayerischen Vereinsbank beruht und in den wenigen Bemerkungen zu den Vorgängen 1938 (ebenda, S.713 u. S. 715) irreführend ist. Die Darstellung der Rolle Reuschs im Konflikt um die BV bei Christian Marx, Paul Reusch und die Gutehoffnungshütte, Göttingen 2013, S. 405-409, ist dagegen instruktiv, wenngleich ebenfalls ohne Quellen aus der BV. 
darunter die Münchner Neuesten Nachrichten ${ }^{30}$. Er engagierte sich in der industriellen Interessenpolitik, war 1927 Initiator der sogenannten Ruhrlade, wurde Vorsitzender der Nordwestlichen Gruppe des Vereins Deutscher Eisen- und Stahlindustrieller und nahm eine Reihe weiterer Funktionen wahr. Von politischen Verirrungen vor 1933 war er keineswegs frei, lehnte er doch die Weimarer Republik entschieden ab. Im Frühjahr 1932 sprach er sich kurzzeitig sogar für eine Zusammenarbeit mit der NSDAP aus, bevor er nach dem von ihm befürworteten Sturz Heinrich Brünings im Frühsommer 1932 die autoritär-antiparlamentarische Präsidialregierung Franz von Papens unterstützte, zu deren Zielen die Beseitigung der Weimarer Verfassungsordnung gehörte. Nach 1933 wuchs die Distanz von Paul Reusch zur NSDAP, deren Antisemitismus er ebenso ablehnte wie deren Wirtschaftspolitik. Trotzdem exponierte er sich in den ersten Jahren nicht sichtbar gegen das NS-Regime.

Aufgrund regimekritischer Äußerungen und nonkonformen Verhaltens wurde Paul Reusch bis 1942 nach und nach aus allen Ämtern vertrieben, so dass er sich auf sein württembergisches Landgut zurückzog ${ }^{31}$. Die von ihm unterzeichneten Briefe während der nationalsozialistischen Diktatur, die sich in den Unterlagen finden, endeten übrigens stets mit normalen Grußformeln und nie mit „Heil Hitler“, im mündlichen Verkehr umging er den Hitler-Gruß oder den „deutschen Gruß“ (der nur vereinzelt begegnet) mithilfe der für den Generaldirektor eines Hüttenwerks üblichen Bergmannssprache und grüßte mit „Glück auf!“ Der Blick auf den Lebensweg und die Aktivitäten von Paul Reusch zeigt, wie schwer seine Persönlichkeit einzuordnen ist; im Aufsichtsrat der Bayerischen Vereinsbank wurde er bis 1938 gerade deshalb mehr und mehr zur Schlüsselfigur, die sich nun anders als 1933 - eindeutig gegen das Regime stellte.

Reusch war seit dem 16. März 1928 Mitglied des Aufsichtsrats der Bayerischen Vereinsbank. Nach dem Tod des mit ihm befreundeten und wirtschaftlich verbundenen Präsidenten des Aufsichtsrats, Dr. Ing. e. h. Fritz Neumeyer, des Generaldirektors der Fritz Neumeyer A.G., wurde Reusch am 1. Oktober 1935 Präsident dieses Gremiums ${ }^{32}$. Im gleichen Jahr wie Fritz Neumeyer verstarb ein weiteres führendes Mitglied des Aufsichtsrats, der Großaktionär der Vereinsbank, Franz von

\footnotetext{
${ }^{30}$ Zur politischen Zielsetzung, die Reusch den Münchner Neuesten Nachrichten Anfang der 1930er Jahre vor allem zur industriellen Interessenvertretung und Formierung einer konservativ-nationalen bürgerlichen Sammlungsbewegung oktroyieren wollte, vgl. die Dokumentation von Kurt Koszyk, Paul Reusch und die „Münchner Neuesten Nachrichten“. Zum Problem Industrie und Presse in der Endphase der Weimarer Republik, in: VfZ 20 (1972), S.75-105, die aufschlussreiche Briefe enthält. Sehr kritisch fällt das Urteil über Reuschs Passivität bei der Gleichschaltung der Zeitung und die Verhaftung ihrer Redakteure aus; vgl. Peter Langer, Paul Reusch und die Gleichschaltung der „Münchner Neuesten Nachrichten“ 1933, in: VfZ 53 (2005), S.203-240.

${ }^{31} \mathrm{Zu}$ den beiden wichtigsten Großunternehmen und die Leitung durch Paul Reusch vgl. Erich Maschke, Es entsteht ein Konzern. Paul Reusch und die Gutehoffnungshütte, Tübingen 1969; jetzt vor allem Marx, Paul Reusch und die Gutehoffnungshütte; Johannes Bähr/Ralf Banken/Thomas Flemming, Die MAN. Eine deutsche Industriegeschichte, München 2008.

${ }^{32}$ Geschäftsbericht für das Geschäftsjahr 1935, S. 2 u. S. 17, in: Historisches Archiv der UniCredit Bank AG München, D-BV-KOM-PUB-67.
} 
Mendelssohn, Mitinhaber der Bankhäuser Mendelssohn \& Co. in Berlin und Mendelssohn \& Co. in Amsterdam. Mendelssohn zählte zu den angesehensten deutschen Bankiers und übte während der Weimarer Republik großen Einfluss in der Finanzwelt aus. Schon seit 1914 war er Präsident der Berliner Industrie- und Handelskammer, von 1921 bis 1933 Präsident des Deutschen Industrie- und Handelstages, daneben Vorsitzender von dessen Ausschuss für das Geld- und Kreditwesen. 1926 wurde Mendelssohn zum Präsidenten der deutschen Gruppe der Internationalen Handelskammer Paris und 1931 zu deren Vizepräsidenten gewählt. Mendelssohn erwarb sich große Verdienste um die Wiederherstellung der internationalen wirtschaftlichen Zusammenarbeit nach dem Ersten Weltkrieg. Nach der Definition der Nürnberger Gesetze von 1935 war Mendelssohn Jude, sein Bankhaus galt als jüdisch, wie auch die spätere „Arisierung“ 1938 zeigt $^{33}$. Gleichwohl waren die ehrenden Bemerkungen des Aufsichtsrats der Bayerischen Vereinsbank am 8. April 1936 in gleichem Ton gehalten wie die für Fritz Neumeyer. So hieß es über Mendelssohn: „Der hochangesehene Verstorbene, der unserem Aufsichtsrate seit dem Jahre 1922 angehörte, stellte seine reiche Erfahrung und klares Urteil mit großer Bereitwilligkeit unserem Institute zur Verfügung und widmete ihm jederzeit sein Interesse. Den beiden hochverdienten Männern schuldet unsere Bank bleibenden Dank; ihr Andenken wird allezeit in Ehren gehalten“34.

Dem aus dem Vorsitz gedrängten Paul Reusch folgte 1938 als Präsident des Aufsichtsrats Kommerzienrat August Bauch (München), der dieses Mandat bis 1950 ausübte. Es ist außerordentlich bemerkenswert, dass die Bayerische Vereinsbank selbst nach der Gleichschaltungsaktion von 1938 einen Parteimann als Vorsitzenden des Aufsichtsrats verhindern konnte, also während der gesamten NS-Zeit keinen Nationalsozialisten an der Spitze hatte, auch wenn Bauchs Resistenz gegen das Regime weniger dezidiert, sein Verhalten vielleicht aus taktischen Gründen „geschmeidiger“ war als das von Reusch. August Bauch hatte bis 1926 als Mitglied des Vorstands gewirkt und gelangte danach in den Aufsichtsrat der Vereinsbank, war also Berufsbankier.

Die drei während der nationalsozialistischen Diktatur amtierenden Aufsichtsratsvorsitzenden (Neumeyer, Reusch und Bauch) gehörten dem Gremium also bereits lange vor 1933 an und waren nicht durch die NSDAP in ihre Funktion gelangt. Wie bei diesen drei Persönlichkeiten gab es aber auch bei anderen Aufsichtsratsmitgliedern eine zum Teil erhebliche Kontinuität über die epochalen Umbrüche von 1933 und 1945 hinweg. Eine wichtige Persönlichkeit im Aufsichtsrat war der Mitinhaber der Mendelssohn-Banken (und 1935 als Nachfolger Franz von Mendelssohns Seniorchef des Unternehmens) Rudolf Löb, der von 1921 bis 1936 diesem Gremium angehörte. Löb führte 1938 die Liquidationsverhandlungen des Bankhauses mit der Deutschen Bank, deren Verhandlungsführer Her-

\footnotetext{
${ }^{33}$ Vgl. Keith Ulrich, Aufstieg und Fall der Privatbankiers. Die wirtschaftliche Bedeutung von 1918 bis 1938, Frankfurt a.M.1998, S. 333 f.

${ }^{34}$ Geschäftsbericht für das Geschäftsjahr 1935, S. 17, in: Historisches Archiv der UniCredit Bank AG München, D-BV-KOM-PUB-67.
} 
mann Josef Abs war ${ }^{35}$. Löb war ein erfahrener und hochverdienter Finanzfachmann, der zwischen 1924 und 1930 verschiedene Reichsregierungen beraten hatte und sogar auf deren Personalpolitik, beispielsweise 1929 bei der Berufung von Hans Schäffer zum Staatssekretär im Finanzministerium, einwirken konnte. 1931 spielte er bei den Verhandlungen zur Lösung der Bankenkrise eine wichtige Rolle. Rudolf Löb war deutscher Jude und emigrierte 1939 über Argentinien in die USA, wo er 1966 in Boston starb ${ }^{36}$. Er zählte (als Mitinhaber der MendelssohnBank und Vertrauter der Familie Mendelssohn) nicht allein zu den bedeutendsten deutschen Privatbankiers, er war darüber hinaus ein eindrucksvoller Repräsentant des deutsch-jüdischen Bildungsbürgertums, herausragender Kunstsammler, hochgebildeter Kenner der antiken Literatur und klassischer Sprachen, aber ausgesprochen polyglott zugleich in den lebenden ${ }^{37}$.

Als Löb unter politischem Druck und im Zuge der „Arisierung“ des Mendelssohn-Aktienpakets auf eine erneute Nominierung verzichtete, kommentierte der Aufsichtsrat dies im März 1938 wie folgt: „Vor der vorjährigen Generalversammlung hat Herr Generalkonsul Rudolf L ö b, der unserem Aufsichtsrate viele Jahre angehört hat, gebeten, von seiner Wiederwahl Abstand zu nehmen. Wir haben mit ihm nicht nur einen hervorragenden Bankfachmann, sondern auch einen wertvollen Berater verloren, der sein kluges Urteil jederzeit in den Dienst unserer Gesellschaft gestellt hat." ${ }^{38}$

Auch hier zeigt sich: Der unter Leitung von Paul Reusch stehende Aufsichtsrat der Bayerischen Vereinsbank hatte sich seine Urteilsfähigkeit bewahrt und scheute noch im Frühjahr 1938 - fünf Jahre nach der Machtergreifung und nahezu drei Jahre nach den antisemitischen Nürnberger Gesetzen - nicht davor zurück, sich ehrend über einen jüdischen Kollegen in einem für eine große Versammlung bestimmten, also nicht geheim zu haltenden, gedruckten Bericht zu äußern, wie er es auch im Falle Franz von Mendelssohns 1936 getan hatte.

Der Vorstand der Bayerischen Vereinsbank wies ebenfalls eine bemerkenswerte Kontinuität auf, obwohl die Nationalsozialisten auch hier wie im Aufsichtsrat versuchten, ihre Personalpolitik durchzusetzen. Durch Verdrängung einzelner Vorstandsmitglieder und Neuberufung einiger anderer gelangen zwar Einbrüche in diese der Politisierung durch die Partei entgegenstehende personelle Kontinuität, aber doch keineswegs mit so nachhaltigem Erfolg, wie es auf den ersten Blick scheinen könnte, bemühten sich doch Aufsichtsrat und Vorstand beharrlich, we-

\footnotetext{
${ }^{35}$ Siehe insgesamt Wilhelm Treue, Das Bankhaus Mendelssohn als Beispiel einer Privatbank im 19. und 20. Jahrhundert, in: Mendelssohn-Studien, Bd.1, Berlin 1972; Lothar Gall, Der Bankier Hermann Josef Abs. Eine Biographie, München 2004, S. 59 ff.; Harold James, Die Deutsche Bank und die „Arisierung“, München 2001, S. 67 ff. u. S. $73 \mathrm{ff}$.

${ }^{36}$ Vgl. Biographisches Handbuch der deutschsprachigen Emigration nach 1933, hrsg. vom Institut für Zeitgeschichte und der Research Foundation for Jewish Immigration unter der Gesamtleitung von Werner Röder und Herbert A. Strauss, Bd. 1, München u. a. 1980, S. 451.

${ }^{37}$ Vgl. den Nachruf von Max Kreutzberger (New York), Rudolf Loeb. Ein König der Privatbankiers, in: Börsen-Zeitung vom 27.8.1966.

${ }^{38}$ Geschäftsbericht 1938, S. 15, in: Historisches Archiv der UniCredit Bank AG München, DBV-KOM-PUB-69.
} 
nigstens eine partielle Autonomie zu bewahren. Eine herausragende Rolle spielte dabei Kommerzienrat Dr. Ing. e.h. Karl Butzengeiger, der dem Vorstand von 1921 bis 1956 (stellvertretend schon ab 1918) angehörte. Bis 1938 in Nürnberg tätig, wurde er 1938 als Primus inter pares des Vorstands der Vereinsbank nach München geholt. Zwar sah das Aktiengesetz von 1937 die Funktion eines Vorstandsvorsitzenden vor ( $\$ 70$, Abs. 2), doch konnten die Banken das Kollegialprinzip beibehalten. In diesem Fall stellte sich die Frage, wer als Sprecher und Repräsentant des Gesamtvorstandes nach außen fungieren und intern die Leitung der Sitzung und andere Koordinationsaufgaben übernehmen sollte: Der Arbeitsausschuss folgte am 28. April 1938 einstimmig dem Vorschlag des Vorstandes, den 56-jährigen Butzengeiger als dienstältestes Vorstandsmitglied mit dieser Leitungsfunktion zu betrauen $^{39}$. Butzengeiger nahm tatsächlich in den kommenden sieben Jahren während des NS-Regimes und im ersten Nachkriegsjahrzehnt eine Schlüsselrolle ein. Danach wechselte er in den Aufsichtsrat und amtierte von 1956 bis 1962 als Stellvertretender Vorsitzender. Insbesondere während der Gleichschaltungsaktion von 1942/43 setzte er sich mit großem Geschick gegen die erneute nationalsozialistische Einflussnahme auf die Vereinsbank zur Wehr.

Der von der Mendelssohn-Bank kommende Friedrich Pasternak war ordentliches Mitglied des Vorstands von 1925 bis 1938. Pasternak, der weitläufig mit dem späteren Literaturnobelpreisträger Boris Pasternak verwandt war, verließ den Vorstand 1938 nach einer Vereinbarung mit der Bank über seine Versorgungsbezüge. Der Druck der Partei gegen „Nichtarier“ im Vorstand der Vereinsbank erreichte in diesem Jahr seinen Höhepunkt und stand im Zusammenhang mit der „Arisierung“ des Mendelssohn-Anteils. Pasternak emigrierte anschließend nach Großbritannien. Der damalige Präsident des Aufsichtsrates, Paul Reusch, schrieb ihm am 4. Februar 1938: „Ich habe mit lebhaftem Bedauern von Ihrem Wunsche, aus dem Vorstand der Bayerischen Vereinsbank auszuscheiden, Kenntnis genommen. Ich will mich unter den obwaltenden Verhältnissen der Erfüllung dieser Bitte nicht weiter widersetzen [...]. Ich behalte mir vor, Ihre Verdienste um die Bank an anderer Stelle entsprechend zu würdigen, es ist mir aber ein Bedürfnis, Ihnen schon heute den aufrichtigsten und herzlichsten Dank für die wertvollen Dienste, die Sie dem Unternehmen in Ihrer langjährigen erfolgreichen Tätigkeit geleistet haben, zum Ausdruck zu bringen. "40 Gemäß einer Vereinbarung mit dem BV-Aufsichtsrat war Pasternak 1933 offenbar auf seinen Wunsch hin bestätigt worden, dass er Anspruch auf ein in monatlichen Raten zu zahlendes Ruhegehalt von jährlich RM 12.000 auf Lebensdauer habe, im Falle seines Ablebens sollte seine Witwe drei Fünftel dieses Betrages erhalten (solange sie nicht wiederverheiratet sei) ${ }^{41}$. In seinem Entschädigungsverfahren nach dem Krieg erklärte Pasternak, dass sich die Bayerische Vereinsbank seiner Entlassung ohne Gefährdung ihrer Existenz

\footnotetext{
${ }^{39}$ Niederschrift über die 47. Sitzung des Arbeitsausschusses der Bayerischen Vereinsbank, 28. 4. 1938, in: Historisches Archiv der UniCredit Bank AG München, D-BV-LO-A-194.

${ }^{40}$ Paul Reusch in einem Schreiben an Pasternak vom 4. 2. 1938, in: Historisches Archiv der UniCredit Bank AG München, D-BV-LO-A-532.

${ }^{41}$ Präsidium des Aufsichtsrats an Bankdirektor Friedrich Pasternak, 16.3.1933, in: Ebenda.
} 
nicht hätte entziehen können ${ }^{42}$. Die Bayerische Vereinsbank erkannte an, dass er seine Stellung ausschließlich wegen seiner Klassifizierung als Jude eingebüßt und deswegen einen Entschädigungsanspruch habe.

Etwa eineinhalb Jahre nach Pasternaks Ausscheiden erörterte der Arbeitsausschuss der Bayerischen Vereinsbank aufgrund einer reichsgerichtlichen Entscheidung am 5. Dezember 1939 dessen Pensionsansprüche an die Bank. Die Sitzungsteilnehmer erklärten: Die reichsgerichtliche Entscheidung kläre nicht eindeutig, ob Nichtarier weiterhin Pensionsansprüche besäßen. Der Vorstand stellte sich zwar auf den Standpunkt, dass „eine Verpflichtung zur Pensionsleistung nicht mehr besteht“. Doch solle Pasternak „lediglich im Wege der freiwilligen Sustentation bis auf weiteres noch RM 300,- monatlich, d. i. der bisher schon an inländische Verwandte des Herrn Pasternak ausbezahlte Betrag, weitergewährt“ wer$\mathrm{den}^{43}$. Damit war die Angelegenheit aber nicht erledigt, weil die Gestapo anfragte, ob die Bayerische Vereinsbank dem früheren Vorstandmitglied Pasternak eine Pension zahle. Der BV-Vorstand antwortete formal korrekt: „Die Anfrage wurde dahin beantwortet, daß der Aufsichtsrat schon vor längerer Zeit den Standpunkt eingenommen habe, daß Herr Pasternak keinen Pensionsanspruch gegen die Bank mehr habe." ${ }^{44}$ Die Zahlungen der Bayerischen Vereinsbank an Pasternaks Verwandte wurden in der Antwort an die Gestapo nicht erwähnt - nur so konnten sie überhaupt erfolgen, weil es schon aufgrund der Devisengesetzgebung nicht möglich war, Überweisungen in das Emigrationsland von Friedrich Pasternak an ihn persönlich zu tätigen. Wie viel bis 1945 tatsächlich gezahlt worden ist, lassen die Unterlagen nicht erkennen. Jedenfalls kam es darüber zum Zerwürfnis zwischen dem Vorstandsmitglied Dietrich und dem früheren Aufsichtsratsvorsitzenden Paul Reusch: Er bezichtigte Dietrich, die von ihm mit Pasternak, Kastl und Mayrhauser getroffenen Vereinbarungen nicht eingehalten zu haben ${ }^{45}$. Nach dem Krieg zahlte die Bayerische Vereinsbank an Pasternak sowohl eine Entschädigung für den Gehaltsausfall bis zum Zeitpunkt, zu dem er regulär als Bankbeamter pensioniert worden wäre, als auch die entsprechenden Ruhestandsbezüge. Allerdings zog sich die endgültige Regelung bis 1961 hin, weil die Bankenaufsicht die durch die Bank gezahlte Entschädigung gemäß der einschlägigen Gesetzeslage als zu hoch bewertete ${ }^{46}$.

Ausschließlich während der NS-Diktatur im Vorstand waren nur drei Personen: von 1936 bis 1938 auf Vorschlag von Paul Reusch der Geheime Regierungsrat a.D.

\footnotetext{
${ }^{42}$ Vormerkung Betr. Entschädigungsansprüche des Herrn Dir. Friedrich Pasternak und des Herrn Dir. Schulmann, in: Historisches Archiv der UniCredit Bank AG München, D-BV-RETA-1498.

43 TOP 4 der Sitzung des Arbeitsausschusses der BV vom 5. 12. 1939, in: Historisches Archiv der UniCredit Bank AG München, D-BV-LO-A-750.

${ }^{44}$ Niederschrift über die 58. Sitzung des Arbeitsausschusses der Bayerischen Vereinsbank vom 5. 12. 1940, in: Historisches Archiv der UniCredit Bank AG München, D-BV-LO-A-195.

${ }^{45}$ Vgl. Akten-Vormerkung vom 29. 7. 1938, in: Historisches Archiv der UniCredit Bank AG München, D-BV-LO-A-782.

${ }^{46}$ Schriftverkehr über die Rechtsansprüche sowie ihrer Berechnungen 1953 bis 1961, in: Historisches Archiv der UniCredit Bank AG München, D-BV-RET-A-1498.
} 
Dr. h.c. Ludwig Kastl, von 1938 bis 1945 Georg Helmreich und von 1938 bis 1945 Bernhard Hoffmann: Die beiden letzteren gelangten nach dem Gleichschaltungsversuch durch die NSDAP in den Bankvorstand. Helmreich und Hoffmann waren zwar Parteigenossen, aber auch qualifizierte Bankfachleute. Helmreich war bis dahin stellvertretendes Vorstandsmitglied und Leiter der Hauptniederlassung Nürnberg der Hypo Bank, Hoffmann Leiter der Hypothekenverwaltung Berlin der Bayerischen Vereinsbank. Bei der schon erwähnten Wahl zum Sprecher des auf vier ordentliche Mitglieder verkleinerten Vorstandes stimmten beide 1938 wie die anderen für den bisherigen Leiter der Hauptniederlassung Nürnberg, Karl Butzengeiger, also nicht für einen Parteimann. Die Berufung von Helmreich behandelte der Vorstand in einem Tagesordnungspunkt, dem man die Überschrift gab: „Interessennahme der NSDAP“. In einem Schreiben des Aufsichtsratsvorsitzenden an den Vizepräsidenten der Reichsbank Kurt Lange wurde Helmreich im Januar 1943 ohne Umschweife der „Vertrauensmann der Partei“ genannt ${ }^{47} .1945$ wurde Helmreich aufgrund eines Befehls der amerikanischen Militärregierung entlassen, später allerdings seine weitere Tätigkeit als unbedenklich bezeichnet. Im Spruchkammerverfahren wurde er am 6. Februar 1947 in die Gruppe IV (Mitläufer) eingestuft, ihm wurde von zahlreichen Entlastungszeugen, darunter jüdischen, in Eidesstattlichen Erklärungen bestätigt, eher unpolitisch gewesen zu sein und sich auch gegenüber „Nicht - Pgs“ kameradschaftlich verhalten zu haben. Mit diesen habe er gemeinsam alle Entscheidungen getroffen, weshalb die Partei mit ihm unzufrieden gewesen sei. Der Versuch von NSDAP-Funktionären, ihn zu verdrängen, habe er jedoch abwehren können ${ }^{48}$. Hoffmann wurde 1939 zur Wehrmacht eingezogen und übte seine Vorstandstätigkeit nur kurzzeitig aus.

Ludwig Kastl war kein Nationalsozialist, er war Jurist und Wirtschaftsfachmann, international erfahren als Diplomat und Kolonialfachmann. Von 1915 bis 1920 fungierte er als Leiter der deutschen Zivilverwaltung in Deutsch-Südwestafrika. Als Ministerialrat im Reichsfinanzministerium war Kastl seit 1921 an den Reparationsverhandlungen beteiligt, die 1924 zum Dawes-Plan und 1929 zum YoungPlan führten. Er war seit 1925 geschäftsführendes Präsidialmitglied des Reichsverbandes der Deutschen Industrie und gehörte von 1929 bis 1932 der Mandatskommission des Völkerbundes in Genf an. Wie es in den „Wirtschaftlichen Privat-Informationen“ von Fritz Wille hieß, war Kastl „nach der Machtübernahme durch den Nationalsozialismus und die dadurch bedingte Zusammenfassung der Wirtschaft“49 aus dem Reichsverband der Deutschen Industrie ausgeschieden und hatte sich 1933 als Rechtsanwalt beim Berliner Kammergericht niedergelassen. Das NS-Regime, dem er kritisch gegenüberstand, verdrängte ihn nach und nach aus allen Funktionen, so auch bei der Bayerischen Vereins-

\footnotetext{
${ }^{47}$ Januar 1943, Kopie, in: Historisches Archiv der UniCredit Bank AG München, D-BV-LOA-788.

${ }^{48}$ Spruchkammerbescheid und weitere Unterlagen, in: Historisches Archiv der UniCredit Bank AG München, D-BV-Per-A-1313.

${ }^{49}$ Heft Nr. 172 vom 17. 12. 1936.
} 
bank. An den neuen Aufsichtsratsvorsitzenden Bauch schrieb er am 17. Juni 1938: „Ich nehme Bezug auf unsere Unterredung am Dienstag, den 14. d. Mts., und erlaube mir, Ihnen mitzuteilen, dass ich angesichts der Entwicklung der Verhältnisse und unter Berücksichtigung der mir von Ihnen gemachten Mitteilungen den Wunsch habe, mit Wirkung vom 30. Juni d. Js. aus dem Vorstand der Bayerischen Vereinsbank auszuscheiden." ${ }^{50}$

\section{Die Gleichschaltungsaktionen gegen die Bayerische Vereinsbank 1938 und 1942/43}

Der geschilderte Personalaustausch im Vorstand der Vereinsbank war Teil der gesamten Gleichschaltungsaktion, die in der Neuaufteilung der Aktienpakete ihr allerdings nur vorläufiges Ende fand. Wie unterschiedlich das Ausscheiden von vier BV-Vorstandsmitgliedern (Pasternak, Kastl, von Mayrhauser, Dietrich) sowie des stellvertretenden Vorstandsmitglieds Falkner auch begründet war - diese Aktion schwächte die Widerstandskraft der Bank erheblich. Keiner der Ausscheidenden war Nationalsozialist, zwei - Pasternak und Kastl - mussten definitiv auf Druck der NSDAP gehen. Das altersbedingte Ausscheiden des inzwischen 69-jährigen Direktors und Vorstandsmitglied Dr. Hans Chr. Dietrich war der Partei sehr willkommen, und von Mayrhauser lieferte zwar möglicherweise durch den Vorwurf eines (geringfügigen) Devisenvergehens einen Vorwand für eine Entlassung; er war aber schon vorher bei der NSDAP nicht wohlgelitten gewesen. Auf eine Anfrage des „Sonderbeauftragten für Wirtschaftsangelegenheiten der Hauptstadt der Bewegung“, Christian Weber, vom 2. Juni 1938 reagierte der Vorsitzende des Aufsichtsrats der Vereinsbank, August Bauch, mit einem Brief, der keinen Zweifel daran lässt, dass die Leitung der Vereinsbank im Frühjahr 1938 unter massivem Druck handelte und eine Reihe personeller Forderungen der NSDAP erfüllen musste, darunter die Entlassung jüdischer Vorstands- und Aufsichtsratsmitglieder: „Das Ausscheiden des Herrn Dr. Kastl aus dem Vorstande stellt einen Bestandteil des Auflagenkomplexes dar, welcher der Verwaltung des Instituts von der Reichsaufsicht durch den für die Bank bestellten Reichskommissar, Herrn Ministerialrat Dr. Leonhard Meukel, Senatspräsident am Oberlandesgericht, München, in den Verhandlungen am 27. und 28. April 1938 bekannt gegeben worden ist. Diese Auflagen waren in ihrer Gesamtheit Ergebnis wiederholter Besprechungen des Herrn Reichskommissars mit dem Stabsleiter des Herrn Reichsschatzmeisters. “51

Der Brief beweist, dass es sich im Falle Kastls nicht um eine personalpolitische Einzelmaßnahme handelte, sondern um eine längere Zeit vorbereitete Aktion ge-

\footnotetext{
${ }^{50}$ Dr. h.c. Ludwig Kastl an Kommerzienrat Bauch, Vorsitzer des Aufsichtsrates, 17.6. 1938, in: Historisches Archiv der UniCredit Bank AG München, D-BV-LO-A-528.

${ }^{51}$ Vorsitzer des Aufsichtsrates an Sonderbeauftragten für Wirtschaftsangelegenheiten der Hauptstadt der Bewegung, 8.6.1938, in: Historisches Archiv der UniCredit Bank AG München, D-BV-LO-A-759.
} 
gen die Autonomie der Vereinsbank, die dem Regime ein Dorn im Auge war ${ }^{52}$. Trotz unvermeidlicher Zugeständnisse hatten sich Vorstand und Aufsichtsrat der Bayerischen Vereinsbank bis dahin nicht gleichschalten lassen, sondern ideologische Elementarprinzipien des Regimes, wie den Antisemitismus, zumindest teilweise unterlaufen. Tatsächlich geriet die Vereinsbank gerade deshalb zunehmend unter politischen Druck, weil sie in den ersten fünf Jahren des Regimes alles andere als nationalsozialistisch war: Ein Beispiel dieser Resistenz bildet die Vorstandstätigkeit von Friedrich Pasternak. Gegen das Ausscheiden des jüdischen Vorstandsmitglieds hatten zwei Großaktionäre bis 1938 entschiedenen und erfolgreichen Widerstand geleistet, die Mendelssohn-Bank und die Gutehoffnungshütte. Ihre Vertreter in Vorstand und Aufsichtsrat schafften es bis 1938, eine Mehrheit gegen die Entlassung Pasternaks zu mobilisieren. Beide Großaktionäre wurden aber 1938 selbst entmachtet, als das Regime den Verkauf des Aktienpakets der Gutehoffnungshütte und den Rücktritt von Paul Reusch als Aufsichtsratsvorsitzendem erzwang und im gleichen Jahr die Liquidierung des deutschen Teils der Mendelssohn-Bank in Berlin betrieb. Ohne Zweifel stellte das Jahr 1938 einen tiefen politischen Einschnitt für die Vereinsbank dar, obwohl dem Regime im politischen oder ideologischen Sinn nach wie vor keine wirkliche Gleichschaltung gelang. Allerdings besaßen danach Juden in der Bayerischen Vereinsbank kein Refugium mehr. So weit ging die Autonomie der Bank dann doch nicht.

Vorstand und Aufsichtsrat wurden außerdem gezwungen, auf mehrere (nichtjüdische) Mitglieder zu verzichten, die zweifellos regimekritisch eingestellt waren. Die Leitung der Bank und ihre Gremien mussten künftig erheblich stärker lavieren als vorher, zumal ihnen „Aufpasser“ aufgezwungen wurden, auch wenn diese keine Parteifunktionäre im engeren Sinne waren. Und schließlich bildete die in Teilen ungleich angepasstere Belegschaft eine „Betriebsöffentlichkeit“, auf die Vorstand und Aufsichtsrat Rücksicht nehmen mussten, weil interne Informationen an Parteistellen gelangten. Ein Beispiel bildete die von den Betriebsobleuten herausgegebene Werkzeitschrift, in der 1938 eine groß aufgemachte Mitteilung an alle Betriebsangehörigen veröffentlicht wurde: „Mit Rundschreiben vom 4. März wurde das Ausscheiden des nichtarischen Vorstandsmitgliedes Pasternak sowie zwei weiteren nichtarischen stellvertretenden Direktoren bekannt gegeben. Es kann auch heute noch hie und da die Beobachtung und Wahrnehmung gemacht werden, dass unser Institut als Judenbank bezeichnet wird [...]. In jedem Falle ist es Pflicht eines jeden Betriebsangehörigen darauf hinzuweisen, dass in unserem Betrieb keinerlei derartige Zusammenhänge mehr gegeben sind; Aufsichtsrat, Vorstandschaft und Gefolgschaft sind vollkommen frei von jüdischen Personen und jüdischem Einfluss." ${ }^{53}$ Im Sommer 1933 hatten der Bayerischen Vereinsbank sieben „Nichtarier“ unter ca. 1600 Mitarbeitern angehört, wie Vor-

52 Vgl. das Memorandum von Aufsichtsrat und Vorstand vom 27.6. 1939, in dem detailliert dargelegt wird, welche Forderungen der Partei die Vereinsbank erfüllt hatte, in: Historisches Archiv der UniCredit Bank AG München, D-BV-KOM-A-1855.

53 „Betrieb und Vertrauen“, Folge 3, in: Historisches Archiv der UniCredit Bank AG München, D-BV-KOM-PUB-968. 
standsmitglied Dr. Dietrich zur „derzeit aktuellen Arierfrage“ berichtete. „Er hielt dafür, dass die Frage für die Bank kaum Bedeutung erlangen werde. " ${ }^{54}$

Wie kam es 1938 zu dieser Wendung? Angesichts der ideologischen Abstinenz der Geschäftsberichte von Vorstand und Aufsichtsrat und der Tatsache, dass noch 1936 mit Paul Reusch ein Aufsichtsratsvorsitzender gewählt wurde, der das NSSystem ablehnte, ist es eher verwunderlich, dass die Bank diesen Kurs so lange durchhalten konnte und jahrelang, zumindest bis 1938, in den Gremien und unter den Mitarbeitern Juden waren. Der Arbeitsausschuss, der aus Vorstands- und Aufsichtsratsmitgliedern bestand, legte am 27. Juni 1939 ein Memorandum über die Vorgänge vor: Es war ganz offensichtlich zur Verteidigung gegen die nationalsozialistische Einflussnahme gedacht, wollte sie anscheinend aber auch ausdrücklich aktenkundig machen. Hierin hieß es unter anderem: „Seit Anfang des Jahres 1938 begannen Verhandlungen über eine G l e i c h s c h a l t u n g der BV. Die Anregung hierzu ging von einer Stelle der Reichsparteileitung in München aus; es nahmen daran teil die - für Gleichschaltungsfragen zuständige - Behörde des Beauftragten für den Vierjahresplan in Berlin [Ministerpräsident Hermann Göring] und das Reichswirtschaftsministerium als Aufsichtsbehörde über die Banken. Die Besprechungen dauerten einige Monate; sie wurden zum Teil auch mit der BV geführt. Den Ausgangspunkt zu dem Vorgehen bildete die Judenfrage in der BV." ${ }^{\text {"5 }}$

In dem Memorandum hieß es weiter: Seit Ende 1935 - also einige Monate nach Erlass der antisemitischen Nürnberger Rassegesetze vom 15. September 1935 hätten „einige Herren des Vorstands und des Aufsichtsrates immer wieder auf die notwendige Lösung: die Entfernung der Juden aus Aufsichtsrat, Vorstand und Gefolgschaft und die Aufhebung der Freundschaftsbeziehungen zu Mendelssohn \& Co., gedrängt “56. Diese Formulierung sollte offensichtlich die Linientreue der Vereinsbank dokumentieren, eine Mehrheit für ein solches Vorgehen bestand dort aber nicht. Auch in der Sitzung des Arbeitsausschusses am 1. Oktober 1935, die dem Erlass der Nürnberger Gesetze folgte, findet sich kein Hinweis auf eine derartige Diskussion, vielmehr ein gegenteiliges Indiz: Bei der Neuwahl des Aufsichtsratspräsidenten schlug der Mitinhaber der Mendelssohn Bank, Löb, die Wahl von Paul Reusch vor: „Diesem Antrag wird von allen Herren zugestimmt [...]. Herr Dr. Dietrich begrüßt den Vorschlag im Namen des Vorstandes. "57 Da Löb „Nichtarier“ und Reusch kein Nationalsozialist war, bei der Sitzung aber drei Reichskommissare, vier Aufsichtsräte und fünf Vorstandsmitglieder anwesend wa-

${ }^{54}$ Protokoll der 877. Sitzung des AR der Bayerischen Vereinsbank 9.6. 1933, S. 4, in: RheinischWestfälisches Wirtschaftsarchiv Köln (künftig: RWWA), 130-400 101-21/7.

${ }_{55}$ Memorandum des Arbeitsausschusses, vom Aufsichtsratsvorsitzenden August Bauch am 27.6. 1939 übersandt und dem Protokoll dieser Sitzung des Arbeitsauschusses beigefügt, in: Historisches Archiv der UniCredit Bank AG München, D-BV-LO-A-716.

${ }^{56}$ Mit den genannten Herren waren u. a. Heinrich Roeckl gemeint; Niederschrift über die 900. Sitzung des Aufsichtsrates der Bayerischen Vereinsbank vom 25.4. 1938, in: Historisches Archiv der UniCredit Bank AG München, DBV-LO-A-208.

${ }^{57}$ Niederschrift über die 35. Sitzung des Arbeitsausschusses der Bayerischen Vereinsbank, 1. 10. 1935, in: Historisches Archiv der UniCredit Bank AG München, D-BV-LO-A-205. 
ren, kann zumindest der Führungszirkel das Ausscheiden jüdischer Mitglieder kaum befürwortet haben.

Wie groß der Druck des Regimes auf die Vereinsbank war, geht aus einem Aktenvermerk hervor, den das Vorstandsmitglied der Bayerischen Handelsbank und Aufsichtsratsmitglied der Vereinsbank (1921-1938) Wilhelm Freiherr von Pechmann am 22. Januar 1936 angefertigt hatte. Darin erwähnte er Gespräche mit dem nationalsozialistischen Oberbürgermeister Karl Fiehler und Gauleiter Adolf Wagner. Beide hatten erklärt, dass die Präsenz nichtarischer Mitglieder im Vorstand und in leitenden Positionen der Bayerischen Vereinsbank ihr schade. Zur möglichen Wahl Robert von Mendelssohns in den Aufsichtsrat erklärte Pechmann: „Wenn in der nächsten Generalversammlung Herr Robert von Mendelssohn an Stelle seines Vaters in den Aufsichtsrat gewählt würde, so wäre dies ein Akt, wie er unter normalen oder nur halbwegs normalen Verhältnissen nicht selbstverständlicher sein könnte. Von solchen Verhältnissen sind wir aber z. Zt. leider weit entfernt [...]. Nach alledem habe ich, obwohl es gerade mir namenlos schwer fiel, dazu raten müssen, die Zuwahl des Herrn Robert von Mendelssohn bis zur Wiederkehr einer weniger überreizten Zeit zurückzustellen." ${ }^{58}$

Allerdings erklärte der Münchner Fabrikant Heinrich Roeckl später in einer Aufsichtsratssitzung am 25. April 1938, die sich mit den Forderungen von Parteistellen an die Vereinsbank befasste: Seit er vor zwei Jahren in den Aufsichtsrat und den Arbeitsausschuss der Bank gewählt worden sei, „seien immer wieder aus Kreisen der Arbeitsfront, der Partei usw. Bedenken und Bemängelungen wegen der in der Verwaltung der Bank an maßgebender Stelle befindlichen Nichtarier an ihn herangetragen worden. Er habe jedes Mal Herrn Dr. Reusch davon in Kenntnis gesetzt und selbst seine starken Bedenken wegen des weiteren Verbleibens dieser Nichtarier in zahlreichen Briefen zum Ausdruck gebracht. Trotzdem er dabei die Unterstützung der Herren Dr. Dietrich und Baron von Pechmann gefunden habe, habe Herr Dr. Reusch insbesondere in der Frage des nichtarischen Vorstandsmitgliedes Pasternak seine Ansicht nicht geändert. Erst infolge davon habe sich dann der Gedanke entwickelt, dass die Partei durch die Gemeindebank ein Aktienpaket erwerben solle, um die Gleichschaltung durchzuführen. “59

Zur Rechtfertigung der Bank gegenüber den Gleichschaltungsinstanzen führte der Arbeitsausschuss in seinem Memorandum vom Juni 1939 an, dass aus „der Gefolgschaft die wenigen (5) beteiligten Angestellten von 1934 bis Ende 1936“ ausgeschieden seien, im November 1936 sei bei Mendelssohn \& Co durchgesetzt worden, dass das 1922 abgeschlossene Freundschaftsverhältnis „durch die Entwicklung der Verhältnisse gegenstandslos geworden“ sei. Bis 1937 seien zwei der bis 1936 drei noch im Aufsichtsrat verbliebenen „nichtarischen Aufsichtsratsmitglieder (1 Herr war vorher von sich aus zurückgetreten) “ ausgeschieden ${ }^{60}$. Es han-

58 RWWA, 130-400 101-21/6.

${ }^{59}$ Niederschrift über die 900. Sitzung des Aufsichtsrates der Bayerischen Vereinsbank vom 25.4. 1938, in: Historisches Archiv der UniCredit Bank AG München, DBV-LO-A-208.

${ }^{60}$ Memorandum des Arbeitsausschusses, vom AR-Vorsitzenden Bauch am 27.6.1939 übersandt und dem Protokoll dieser Sitzung des Arbeitsausschusses beigefügt, S. 2, in: Histori- 
delte sich wohl um die Mitglieder Dr. Karl Freiherr von Hirsch und Dr. Johann Jakob Schulmann.

Karl von Hirsch entstammte einer alten jüdischen Bankiersfamilie, die zu den frühesten in Bayern geadelten Juden gehörte. Ein Mitglied der Familie, Josef von Hirsch, Mitgründer der Süd-Chemie (1805-1885), ließ sich später in Planegg bei München als Grundbesitzer nieder. Die Familie Hirsch, die aus Würzburg stammte, wo schon am Beginn des 19. Jahrhunderts Jakob von Hirsch (17651840) zum wichtigsten Hofbankier geworden war, hatte an der Gründung sowohl der Hypo-Bank (durch Joel Jacob von Hirsch und Samuel von Hirsch) als auch der Vereinsbank (durch Emil von Hirsch) mitgewirkt. 1885 übernahm die BV das Bankhaus Hirsch. Die Familie von Hirsch - von der einige Angehörige katholisch wurden, andere jüdisch blieben - besaß also über Generationen hinweg enge persönliche und geschäftliche Bindungen an die Vereinsbank und die Hypo-Bank. Karl von Hirsch erlitt das schrecklichste Schicksal unter den jüdischen Gremienmitgliedern der Bayerischen Vereinsbank: Gemeinsam mit seinem Bruder Rudolf wurde er in das KZ Theresienstadt deportiert, wo er 1944 starb.

In dem Rechenschaftsmemorandum der Vereinsbank von 1939 wurde außerdem erwähnt, dass für die Mendelssohn-Bank ein ,arischer"Vertreter (Paul Stach) gewählt worden sei. „Zu den Maßnahmen bis hierher wurde eine einheitliche Stellung innerhalb der Verwaltung der BV unter Überwindung mancher Hemmungen erreicht". Diese Formulierung ist so gewunden, dass man ihr die internen Schwierigkeiten ebenso anmerkt wie die mangelnde Zustimmung zur nationalsozialistischen Gleichschaltung. Verblieben waren „als Nichtarier“ bis 1938 noch das Vorstandsmitglied Pasternak sowie zwei Prokuristen mit dem Titel „stellvertretende Direktoren“.

Was aus diesen beiden Prokuristen wurde, ist bisher nicht bekannt. Auch die Frage, ob die fünf „nichtarischen“ Mitarbeiter zwischen 1934 und Ende 1936 aus Alters- oder anderen normalen Gründen ausschieden oder ob sie dazu gedrängt oder entlassen wurden, muss offen bleiben. Letzteres erscheint aus mehreren Gründen unwahrscheinlich: Zum einen hat der BV-Vorstand verschiedentlich noch Ende der 1930er und zu Beginn der 1940er Jahre darauf hingewiesen, dass Entlassungen bei der Bank seit den Aufbaujahren 1923/24 wenn überhaupt, ausschließlich dann erfolgt seien, wenn strafrechtlich zu ahndende Vergehen vorgelegen hätten. Zum anderen ist zu berücksichtigen, dass die Bank bis 1936/37 drei jüdische Aufsichtsratsmitglieder und bis 1938 mindestens ein jüdisches Vorstandsmitglied und zwei Prokuristen hatte. Sollte da ein einfacher Mitarbeiter ausschließlich deshalb entlassen werden, weil das Regime ihn als „Nichtarier“ eingestuft hatte? Gerade weil die Bayerische Vereinsbank sich auch in dieser Hinsicht nicht systemkonform verhielt, bekam sie 1938 Schwierigkeiten.

Im Übrigen, so das Memorandum von 1939, sei angesichts der Mehrheitsverhältnisse im Aufsichtsrat mit den beiden Großaktionären Gutehoffnungshütte und Mendelssohn-Bank bis 1938 keine Änderung möglich gewesen. Zwar war das nicht die ganze Wahrheit, weil beide jeweils nur 10 Prozent des Aktienkapitals der

sches Archiv der UniCredit Bank AG München, DBV-LO-A-716. 
Vereinsbank hielten, sie also noch mit anderen Anteilseignern - darunter der Familie Neumeyer, die Kolbermoor-Union, seit 1937 auch August Baron von Finck - übereingestimmt haben müssen, um eine Mehrheit zu erreichen. Tatsächlich lag hier aber doch ein wesentlicher Grund: Solange sich um diese beiden Großaktionäre herum - also „arische“ und „nicht-arische“ in der Sprache des NS-Regimes - mit einer durchsetzungsstarken systemkritischen Persönlichkeit wie Paul Reusch als Aufsichtsrats-Präsidenten eine resistente Führung halten konnte, gelang es dem NS-Regime nicht, seine antisemitische Politik restlos durchzusetzen. In keinem Fall war dies so geräuschlos möglich, wie es die NSDAP im Bankwesen bis zur weiteren Radikalisierung des Antisemitismus 1937/38 wünschte.

Aus diesem Grund ging die Partei zunächst gegen den widerspenstigen Aufsichtsratsvorsitzenden Paul Reusch und die Gutehoffnungshütte vor, die sich bis dahin erfolgreich dem Druck der Nationalsozialisten widersetzt hatten. Bei Reusch handelte sich um den schwierigeren Gegner, dem mit dem Instrumentarium des Staatsantisemitismus nicht beizukommen war; schließlich war Reusch auch kein „Linker“, sondern ein deutschnationaler Gegner der Weimarer Republik und in der Schwerindustrie des Ruhrgebiets fest verwurzelt. Darauf nahm das Regime aber seit 1938 keine Rücksicht mehr. So hieß es in dem Memorandum des Vorstands vom 27. Juni 1939: „Gleichzeitig mit der Gleichschaltungsfrage lief das Verlangen nach einem Besitzwechsel in dem bei der Gutehoffnungshütte befindlichen Pakete an BV.- Aktien, das rund RM 3 Millionen Aktien $=$ rund 10\% des Aktienkapitales der BV. betrug. Der Vorstand der Gutehoffnungshütte wurde Anfang März 1938 aufgefordert, diesen Aktienbesitz abzugeben und fügte sich im April 1938 durch den Verkauf an ein bayerisches öffentlichrechtliches Geldinstitut, wobei damals die Frage offen blieb, ob dieses Institut die Aktien für seinen eigenen Besitz oder als Treuhänderin für eine andere Stelle erwarb." ${ }^{11}$

Tatsächlich hatte Hermann Göring von Paul Reusch den sofortigen Verkauf des BV-Aktienpakets mit der Absicht verlangt, es der NSDAP zu übereignen. Diese Transaktion sollte offenbar auf einem Umweg erfolgen, damit der auf einen ,arischen“ Kapitaleigner dieses Formats ausgeübte Zwang nicht allzu deutlich wurde: Wohl um nach außen hin die Form zu wahren, ordnete der Reichsschatzmeister der NSDAP, Franz Xaver Schwarz, die Übernahme des Pakets durch die Bayerische Gemeindebank an ${ }^{62}$ - ein im übrigen im NS-System oftmals praktiziertes zweistufiges Verfahren, das selbst bei umfangreichen „Arisierungen“ zuweilen angewandt wurde.

In der Aufsichtsratssitzung der Bayerischen Gemeindebank wurde am 28. Oktober 1938 mitgeteilt: Der Reichsschatzmeister der NSDAP habe schriftlich den Erwerb eines Aktienpakets der Bayerischen Vereinsbank beantragt. Dies entspreche dem Wunsch des Generalfeldmarschalls Göring. Wenn die Gemeindebank „auch formell als Besitzer der Aktien in Erscheinung tritt, so hat sie doch lediglich

\footnotetext{
61 Ebenda.

${ }^{62}$ Heinrich VII. Reuss, Bayerische Vereinsbank, unveröff. Dokumentation, S. 8, in: Historisches Archiv der UniCredit Bank AG München, D-BV-KOM-A-1585.
} 
als Beauftragte dritter Stellen gehandelt. Gauleiter Wagner hat gleichfalls sein Interesse an dieser Angelegenheit bekundet. “63

So reibungslos wie es hier erschien, verlief die Transaktion des Aktienpakets der Gutehoffnungshütte indes nicht, weil Reusch zunächst versuchte, den Verkauf zu verhindern. Er selbst erreichte eine Unterredung mit Hermann Göring, die aber offenbar nicht das gewünschte Ergebnis brachte. Auf Reuschs Bitte intervenierte dann der Münchner Bankier August von Finck ${ }^{64}$, allerdings ebenfalls erfolglos, wie ein Referent des Bayerischen Wirtschaftsministeriums notierte: Göring „habe [...] nach kurzem Vortrag erklärt, dass er z.Z. mit wichtigen Arbeiten überlastet sei“ und dass von Finck sich an die „Münchner Stelle“ wenden möge. Nach mehrfachen Verhandlungen in München sei von Finck am 15. März 1938 erneut nach Berlin gefahren ${ }^{65}$. Doch offenbar erwiesen sich auch diese Versuche Fincks (der NSDAP-Mitglied war) als Sackgasse, teilte doch der Reichskommissar der Bayerischen Vereinsbank, Dr. Meukel, am 25. März 1938 fernmündlich mit, dass Generaldirektor Reusch den Vorsitz im Aufsichtsrat niedergelegt und Bankier von Finck ,sein Mandat, zwischen der Leitung und dem Aufsichtsrat der Bank einerseits und den Berliner Stellen andererseits zu vermitteln, wegen Aussichtslosigkeit zurückgegeben“ habe ${ }^{66}$. In der Aufsichtsratssitzung vom 29. März teilte Finck selbst mit, „daß die NSDAP auf einer Interessennahme an der Bayerischen Vereinsbank bestehe, nicht wegen eines Geldbedarfes, sondern aus dem Wunsche und Interesse, die Gleichschaltung der Bank durchzuführen"67.

Etwas undurchsichtig bleibt die zeitweilige Absicht Fincks, einen Teil des Pakets von Reusch zu übernehmen, um es der NSDAP zu entziehen. Sicher hat auch diese Aktion ihm den Unwillen der Partei eingetragen, doch ist nicht erkennbar, ob er darüber mit Paul Reusch gesprochen hat ${ }^{68}$. Die Vereinsbank selbst besaß keinerlei Einfluss auf diese Transaktion, dies zeigt die eher beiläufig gegebene, aber wesentliche Anspielungen enthaltende Information des Vorstands an das 1937 als „arischer“ Vertreter des Mendelssohn-Aktienpakets gewählte Aufsichtsratsmitglied Paul Stach, die wohl auch als Warnung hinsichtlich des MendelssohnAnteils gemeint war: „Zu Ihrer Information fügen wir bei, daß das Aktienpaket

\footnotetext{
${ }^{63}$ Niederschrift über die Sitzung des Aufsichtsrats der Bayerischen Gemeindebank, 28. 10. 1938, in: Archiv der Bayern LB, Bestand Bayerische Gemeindebank, Akte Nr. 201.

${ }^{64}$ Niederschrift der 899. Sitzung des Aufsichtsrats der BV vom 29.3.1938, TOP 1, in: Historisches Archiv der UniCredit Bank AG München, D-BV-LO-A-207. Zu Finck vgl. auch Gerald D. Feldman, Die Allianz und die deutsche Versicherungswirtschaft 1933-1945, München 2001.

${ }^{65}$ Vormerkung des Bayerischen Staatsministeriums für Wirtschaft vom 17.3.1938, in: Bayerisches Hauptstaatsarchiv München, Allg. StA MWi 317.

${ }^{66}$ Vormerkung des Bayerischen Staatsministeriums für Wirtschaft vom 25.3. 1938, in: Ebenda.

${ }^{67}$ Niederschrift der 899. Sitzung des Aufsichtsrats der BV vom 29.3.1938, TOP 1, in: Historisches Archiv der UniCredit Bank AG München, D-BV-LO-A-207.

${ }^{68}$ Vgl. dazu die Besprechung zwischen von Finck und von Thelemann mit Vertretern von Vorstand und Aufsichtsrat der Bayerischen Vereinsbank am 10.3.1950, in: Historisches Archiv der UniCredit Bank AG München, D-BV-KOM-A-1585, Heinrich VII. Reuss, Bayerische Vereinsbank, unveröff. Dokumentation, S. 8 u. S. 32.
} 
der GHH (Gutehoffnungshütte) inzwischen an die Bayerische Gemeindebank, München - wie uns gesagt wurde - als Treuhänderin verkauft worden ist." ${ }^{\text {"69 }}$

Es ist aufschlussreich, dass 1938 nahezu parallel jüdische und nichtjüdische Kapitaleigner durch politischen Druck gezwungen wurden, ihre Aktienpakete zu verkaufen: Das Mendelssohn-Paket hatte auf dem Höchststand während der ersten fünf Jahre des NS-Regimes zeitweilig einen Wert von 5.707 Mio. RM, 1938 wurde es noch mit 3.282.900 Mio. RM bewertet, wie Paul Stach, Miteigentümer der Mendelssohn-Bank Amsterdam, mitteilte ${ }^{70}$. Das BV-Aktienpaket war also vom Berliner Sitz Mendelssohns auf das Amsterdamer Haus transferiert worden. Kastl und Pasternak arrangierten dann im Dezember 1938 den Verkauf der Anteile an die Bank Merck, Finck \& Co., kurz bevor das Amsterdamer Haus der Bank Mendelssohn zusammenbrach. Allem Anschein nach ist der Verkauf selbst korrekt abgewickelt worden, anders als die korrespondierende Liquidierung der Mendelssohn-Bank in Berlin, wenngleich alle Ereignisse (Verkauf des Vereinsbank-Pakets, „Arisierung“ des Bankhauses in Berlin, Zusammenbruch des Bankhauses in Amsterdam) in das Jahr 1938 fielen.

Tatsächlich hatte die Vereinsbank weder den Verkauf des Aktienpakets der Gutehoffnungshütte, noch die „Arisierung“ der Mendelssohn-Bank betrieben bzw. durchgeführt. Sie hat vielmehr diesen erzwungenen Verkauf als einen Schlag gegen ihre Unabhängigkeit angesehen, und die Befürchtung weiterer Eingriffe klingt in der fast beschwörenden Formulierung ihres Memorandums mit: „Bei dem Abschluß der Verhandlungen wurde durch die diese Verhandlungen führende Parteistelle die Zusicherung gegeben, daß mit dem Programme die Aktion gegen die BV (!) erledigt und ein weiteres Eingreifen in die Selbständigkeit der Bank nicht beabsichtigt sei. Die BV. solle ihren privatwirtschaftlichen Charakter behalten, weder die Funktion einer Art von Partei-Bank noch eines von öffentlichrechtlichen Körperschaften abhängigen Institutes erhalten."71

Mit dem Doppelschlag gegen Reusch und Mendelssohn konnte die NSDAP nun auch ihre Personalstrategie energischer verfolgen: Nach der Ausbootung der Gutehoffnungshütte konnte man zugleich ihren systemkritischen Repräsentanten an der Spitze des BV-Aufsichtsrats loswerden und den durch Reusch in den Vorstand gebrachten Ludwig Kastl gleich mit. Und nach der Hinausdrängung von Mendelssohn gab es keinen Grund mehr, seine Vertreter in Vorstand und Aufsichtsrat der Bayerischen Vereinsbank zu dulden: Auf diese Weise war die sogenannte Judenfrage der Bank „gelöst“.

Die Gleichschaltungsaktion im Frühjahr 1938 führte schließlich zum Ausscheiden von insgesamt zehn Aufsichtsrats-Mitgliedern, von den seit 1938 neu in das Gremium gewählten acht Mitgliedern waren sieben Parteimitglieder. Im Auf-

${ }^{69}$ Vorstand an Paul Stach, Amsterdam, 19.4. 1938, in: Historisches Archiv der UniCredit Bank AG München, D-BV-LO-A-744 (Kopie).

${ }^{70}$ Briefwechsel von Bankdirektor Dr. Dietrich und Paul Stach, Amsterdam, vom 8. und 11.4. 1938, in: Historisches Archiv der UniCredit Bank AG München, D-BV-LO-A-78.

${ }^{71}$ Memorandum des Arbeitsausschusses, vom AR-Vorsitzenden Bauch am 27.6.1939 übersandt und dem Protokoll dieser Sitzung des Arbeitsausschusses beigefügt, S. 2, in: Historisches Archiv der UniCredit Bank AG München, D-BV-LO-A-716. 
sichtsrat gab es zur Zeit des nächsten Interventionsversuchs 1942/43 unter den 18 Aufsichtsräten zehn NSDAP-Mitglieder, darunter August von Finck, der Landesobmann der Wirtschaftsgruppe für den Bezirk Bayern war, Willy Messerschmitt, Justizrat Helmuth Schieck (München) sowie Carl Tabel (Creußen).

Im vierköpfigen Vorstand, zu dem noch sechs stellvertretende Mitglieder kamen, über deren parteipolitische Orientierung den Unterlagen - außer im Falle des Betriebsführers Kronawitter - nichts zu entnehmen ist, befanden sich die zwei schon erwähnten Parteimitglieder, von denen eines, nämlich Georg Helmreich immer wieder als Vertrauensmann der Partei bezeichnet wird, ohne dass eine spezifisch parteipolitische Aktivität zu erkennen wäre ${ }^{72}$.

Tatsächlich handelte es sich nun im Vergleich zur Zeit bis 1937/1938 um einen deutlichen Personalwechsel, wobei die nationalsozialistische Zielrichtung nicht zu verkennen ist. Andererseits hat sich das Mehrheitsverhältnis von zehn Parteimitgliedern zu acht Parteiungebundenen im BV-Aufsichtsrat kaum zugunsten der Partei instrumentalisieren lassen. Das zeigt die Tatsache, dass der nach 1945 als unbelastet eingestufte August Bauch von 1938 bis 1950 Aufsichtsratsvorsitzender blieb. Auch die führenden Vorstandsmitglieder Karl Butzengeiger und Dr. Wilhelm Biber waren keine NSDAP-Mitglieder und konnten deshalb nach 1945 bis 1956 bzw. 1959 weiteramtieren. Sie setzten sich, wie die Unterlagen zeigen, immer wieder gegen Gleichschaltungsmaßnahmen durch den Vizepräsidenten der Reichsbank, den NSDAP-Schatzmeister Franz Xaver Schwarz und dessen Stabsführer, den SS-Brigadeführer Hans Saupert, zur Wehr. Auch wenn Vorstand und Aufsichtsrat häufig beteuerten, dass sie die „politische Ausrichtung [...] bereits im Jahre 1938 auf Initiative höchster Parteistellen, nämlich des Herrn Reichsschatzmeisters [der NSDAP, Franz Xaver Schwarz] und des Beauftragten für den Vierjahresplan [Hermann Göring], durchgeführt" hätten, traf die misstrauische Einschätzung der nationalsozialistischen Funktionäre zu: Tatsächlich war die Vereinsbank noch nicht auf dem von ihnen gewünschten systemkonformen Kurs, wie die 1942 und 1943 erneuerten Aktionen von NS-Funktionären gegen die Leitung der Bank belegen.

Selbst der Rest an Autonomie, den sich die Vereinsbank noch bewahrt hatte, störte das NS-Regime. Zunächst suchte der Ministerialdirektor im Reichswirtschaftsministerium, Dr. Joachim Riehle - der ursprünglich für einen Vorstandsposten der Hypo-Bank im Gespräch war - am 25. August 1942 die Vorstandsmitglieder Butzengeiger und Dr. Biber auf und unterrichtete sie über aktuelle bankpolitische Richtlinien des NS-Regimes. Das Reichswirtschaftsministerium wolle, dass die ständigen Angriffe von Parteistellen gegen die Banken aufhörten, dafür müssten jedoch die Angriffsflächen beseitigt werden, die die Banken immer noch böten. Dafür spiele die notwendige Bankenrationalisierung ebenso

\footnotetext{
${ }^{72}$ Vgl. Entwurf eines Briefes des Vorstands der BV an Vizepräsident Lange (Berlin), vermutlich Januar 1943, in: Historisches Archiv der UniCredit Bank AG München, D-BV-LO-A-788; Heinrich VII. Reuss, Vereinsbank - Das Entstehen einer Bankengruppe, München 1994, S. 8; vgl. auch unveröff. Dokumentation von Heinrich VII. Reuss, Bayerische Vereinsbank, S. 146 ff., in: Historisches Archiv der UniCredit Bank AG München, D-BV-KOM-A-1585.
} 
eine Rolle wie die Einschätzung, „dass seitens der Bank der Kontakt mit den maßgebenden Parteistellen nicht in dem erwünschten Mass gepflegt werde. Es genüge nicht, dass man mit einem Herrn der Partei gelegentlich ein Glas Bier trinke, vielmehr sei es notwendig, dass ein offizieller Vertrauensmann der Partei im Aufsichtsrat sei, durch den die Bank jederzeit die Meinung der maßgebenden Parteistellen erholen [gemeint ist wohl: einholen] könne und der auch seinerseits für die Bank eintrete."73

Nach Riehles Vorstoß ergriff auch der Vizepräsident der Reichsbank, Kurt Lange, gegenüber der Vereinsbank die Initiative und beorderte für den 22. Januar 1943 einige Vorstands- und Aufsichtsratsmitglieder zu einem Gespräch in die Industrie- und Handelskammer in München. In Gegenwart weiterer Vertreter des Regimes, unter anderem eines Repräsentanten des Reichswirtschaftsministeriums, erläuterte er ihnen in einem zweistündigen Gespräch - das er weitgehend allein bestritt - die Ziele Hitlers: Es „sei der Wunsch des Führers, die deutschen Banken - einschließlich der Staatsbanken und der großen Privatbankfirmen noch mehr als bisher nationalsozialistisch ausgerichtet zu sehen und zwar dadurch, daß sich Vorstand und Aufsichtsrat durch die Berufung von Persönlichkeiten ergänzen, die in maßgebenden Umfang branchenkundig und gleichzeitig fest in der Partei verankert sind und dadurch den maßgebenden politischen Stellen die erforderliche Gewähr bieten."

Die Drohung war unmissverständlich: Wenn sich eine Bank „spreize“, so sei man genötigt, „bei dem widerstrebenden Institut einen Reichskommissar einzusetzen"74 - dies wäre dann nicht mehr der schon erwähnte klassische Typus eines Reichskommissars - also ein Beamter aus dem zuständigen Fachressort -, sondern ein Parteimann mit außerordentlichen Vollmachten gewesen.

Trotz solcher massiver Pressionen gelang es der Bayerischen Vereinsbank, die Aufnahme des Oberbereichsleiters und Gauhauptamtsleiters Dr. Hans Buchner in den Aufsichtsrat abzuwehren: Man wählte ihn zwar, akzeptierte aber nicht seine Bedingung, weitreichende Vollmachten zu erhalten. Der Aufsichtsratsvorsitzende August Bauch teilte ihm mit: „Nach einhelliger Meinung des Aufsichtsrats lassen sich die von Ihnen neuerdings gestellten Bedingungen für die Annahme Ihrer Wahl, sowie deren Begründung, weder mit der derzeit geltenden Wirtschaftsordnung noch mit dem Aktienrecht, noch mit der Satzung unseres Instituts in Einklang bringen. Der Aufsichtsrat sieht sich deshalb leider nicht in der Lage, die von Ihnen gestellten Bedingungen zu erfüllen." ${ }^{75}$ Tatsächlich handelte Bauch mit

${ }^{73}$ Vormerkung über einen Besuch des Ministerialdirektors Dr. Riehle, Reichswirtschaftsministerium, am 25.8.1942 bei Kommerzienrat Butzengeiger und Dr. Biber, S. $1 \mathrm{f}$, in: Historisches Archiv der UniCredit Bank AG München, D-BV-LO-A-779; vgl. auch Heinrich VII. Reuss, Bayerische Vereinsbank, unveröff. Dokumentation, S. 136 D, in: Historisches Archiv der UniCredit Bank AG München, D-BV-KOM-A-1585.

${ }^{74}$ Besprechungsprotokoll „Parteipolitische Ausrichtung der Banken“, 23. 1. 1943, in: Ebenda, S. 143 D u. S. 144 D.

${ }^{75}$ Vorsitzer des Aufsichtsrates, August Bauch, an Dr. Hans Buchner. Niederschrift der 924. Sitzung des Aufsichtsrates der Bayerischen Vereinsbank, 17.4. 1943, in: Historisches Archiv der UniCredit Bank AG München, D-BV-LO-A-210. 
voller Rückendeckung seines Aufsichtsrats, der einstimmig - also mit den der NSDAP angehörenden Mitgliedern sowie den drei anwesenden Vorstandsmitgliedern, darunter dem „Vertrauensmann“ der NSDAP Helmreich - die Erfüllung der Forderungen Buchners ablehnte und ihn dadurch zum Rückzug veranlasste. Zusätzlich begründete die Vereinsbank diese Ablehnung mit der angesichts der Wirtschaftslenkung des totalitären Regimes bemerkenswerten Feststellung, dass die Bayerische Vereinsbank andernfalls „ihres Charakters als Unternehmen der Privatwirtschaft entkleidet werde" ${ }^{\text {"76 }}$.

\section{Fazit}

Es ist bemerkenswert, in welchem Maße sogar NSDAP-Mitglieder in Vorstand und Aufsichtsrat angesichts der sich verstärkenden Pressionen des Regimes sich über Jahre hinweg für die Bankloyalität anstelle der Systemloyalität entschieden. Differenzen innerhalb der Gremien bestanden zwischen 1936 und 1938 vor allem in Bezug auf die Frage, ob und in welchem Maße sich die Bank von den antijüdischen Maßnahmen des NS-Regimes und seiner rassistischen Ideologie frei halten könne. Selbst diejenigen, die wie der später als Mitläufer eingestufte Heinrich Roeckl oder Freiherr von Pechmann den Widerstand dagegen als aussichtslos ansahen, haben die Attacken auf ihre jüdischen Kollegen in den Gremien nicht befürwortet, sondern mit dem Interesse der Bank argumentiert. Aus den Gremien wurde die nationalsozialistische Ideologie weitgehend verbannt. Selbst die Rolle des mit Hitler, Göring und anderen NS-Funktionären gut bekannten Parteimitglieds August von Finck in der Bank ist nicht eindeutig systemkonform, wie sein Verhalten gegenüber Reusch, aber auch der Mendelssohn-Bank zeigt: Indem er deren Anteil nach Absprache mit deren Vertretern - unter anderem mit Friedrich Pasternak - aufkaufte, kam er einer Arisierung in letzter Minute zuvor. Die Maßnahmen gegen die beiden Großaktionäre im Jahr 1938 konnte die Bank selbst nicht verhindern, sie besiegelten mit dem Ausscheiden von Reusch und Mendelssohn auch das Ende der Präsenz jüdischer Aufsichtsrats- und Vorstandsmitglieder. Hier lag zweifelsfrei die Grenze für die Autonomiebestrebungen der Bank, die durch das Regime und seine Repressionsmechanismen gezogen wurde: Dagegen erwies sich selbst ein einst mächtiger „Ruhrbaron“ wie Reusch als machtlos. Hatten ihn 1932 noch Hitler und Himmler in der Gutehoffnungshütte aufgesucht und wagte es Reusch sogar noch am 5. Februar 1938, eine Einladung Hitlers abzulehnen $^{77}$, so hatte wenige Wochen später Göring keine Zeit mehr für ein Gespräch mit Reusch. Immerhin gelang es dem Vorstand und dem Aufsichtsrat aber auch nach 1938 immer wieder, durch geschicktes Lavieren und Insistieren auf juristischen Formalien die Übernahme der Leitung durch NS-Funktionäre zu verhin-

\footnotetext{
${ }^{76}$ Niederschrift der 924. Sitzung des Aufsichtsrates der Bayerischen Vereinsbank, 17.4. 1943, in: Ebenda.

${ }^{77}$ Büro Reusch an die Präsidialkanzlei des Führers und Reichskanzlers, 5. 2. 1938, zit. bei Marx, Paul Reusch und die Gutehoffnungshütte, S. 409. Bereits 1935 hatte Reusch eine Einladung zum Nürnberger Parteitag der NSDAP nicht angenommen.
} 
32 Aufsätze

dern und bis zum Ende des Regimes eine - wenn auch nur begrenzte - Autonomie zu bewahren. Insofern ist die bis 1938 relativ erfolgreiche und danach jedenfalls nicht ganz erfolglose Hinhaltetaktik ein Beispiel dafür, dass Resistenz gegenüber dem totalitären Anspruch des Regimes möglich war und sich lohnen konnte. 